\title{
Mirror symmetry, Langlands duality, and the Hitchin system
}

\author{
Tamás Hausel \\ Department of Mathematics, University of California, Berkeley, Calif. 94720 \\ Michael Thaddeus \\ Department of Mathematics, Columbia University, New York, N.Y. 10027
}

\begin{abstract}
Among the major mathematical approaches to mirror symmetry are those of Batyrev-Borisov and Strominger-Yau-Zaslow (SYZ). The first is explicit and amenable to computation but is not clearly related to the physical motivation; the second is the opposite. Furthermore, it is far from obvious that mirror partners in one sense will also be mirror partners in the other. This paper concerns a class of examples that can be shown to satisfy the requirements of SYZ, but whose Hodge numbers are also equal. This provides significant evidence in support of SYZ. Moreover, the examples are of great interest in their own right: they are spaces of flat $\mathrm{SL}_{r}$-connections on a smooth curve. The mirror is the corresponding space for the Langlands dual group $\mathrm{PGL}_{r}$. These examples therefore throw a bridge from mirror symmetry to the duality theory of Lie groups and, more broadly, to the geometric Langlands program.
\end{abstract}

When it emerged in the early 1990s, mirror symmetry was an aspect of theoretical physics, and specifically a duality between quantum field theories. Since then, many people have tried to place it on a mathematical foundation. Their labors have built up a fascinating but somewhat unruly subject. It describes some sort of relation between pairs of CalabiYau spaces, but there are several quite different formulations of this relation, with no strong links between them. Notable among these are the toric approach of Batyrev-Borisov [4, 5], leading to a very large class of examples whose Hodge numbers behave as desired, and the symplectic approach of Strominger-Yau-Zaslow [38], hereinafter SYZ. The latter is inspired by the original physics, and holds out the remarkable promise of connecting mirror symmetry to the theory of integrable systems. But it is extremely difficult to find examples.

This paper aims to describe certain pairs of Calabi-Yaus - namely, moduli spaces of flat connections on a curve — which exhibit mirror symmetry phenomena in two different senses: first, they satisfy the requirements of SYZ, and second, their Hodge numbers behave more or less as expected. As far as we know, these are the first non-trivial examples of SYZ mirror partners of dimension greater than 2, so they significantly corroborate the SYZ theory.

Furthermore, our examples relate mirror symmetry to another one of the great dualities of mathematics: the Langlands duality on Lie groups. If $\hat{G}$ is the Langlands dual of a reductive group $G$, then the pairs we study are spaces of flat connections on the same base curve with structure groups $G$ and $\hat{G}$. These spaces are basic objects of study in

T.H. supported by a Miller Research Fellowship at the University of California, Berkeley, 1999-2002. M.T. supported by NSF grants DMS-98-08529 and DMS-00-99688. 
the geometric Langlands program, which has many possible points of contact with mirror symmetry. (For example, although we do not discuss it here, equivalence of derived categories of coherent sheaves plays a prominent part in both.) In the present paper we confine ourselves throughout to the case $G=\mathrm{SL}_{r}$, and ultimately to the case $G=\mathrm{SL}_{2}$ or $\mathrm{SL}_{3}$, but we hope and expect that the mirror relationship holds more generally.

The original reason for suspecting that our moduli spaces might be mirror partners was that they comprise dual pairs of hyperkähler integrable systems. The hyperkähler metric and the collection of Poisson-commuting functions determining the integrable system were constructed in two seminal papers of Hitchin in the late 1980s [23, 24. These structures automatically produce a family of special Lagrangian tori on the moduli spaces, which is a key requirement of SYZ. Moreover, the families of tori on the $\mathrm{SL}_{r}$ and $\mathrm{PGL}_{r}$ moduli spaces are dual in the appropriate sense, which is the other requirement of SYZ. The only tricky point is to extend this story to the moduli spaces of bundles of nonzero degree $d$, which are technically much easier to deal with when $d$ and $r$ are coprime.

To deal with this "twisted" case, our moduli spaces alone are not enough: they must be endowed with extra structures, which physicists call $B$-fields and mathematicians call flat unitary gerbes. These appear whenever mirror symmetry is formulated in sufficient generality. In our case they arise in a particularly natural way, and indeed they are necessary for things to work properly when the degree is nonzero. For instance, as we see in $\$$, our case satisfies not the original formulation of SYZ, but rather an extension proposed by Hitchin 26] to Calabi-Yaus with $B$-fields, of which no examples were previously known. Likewise, the Hodge numbers in our case must be evaluated in a generalized sense involving the $B$ field. We explain in $\$$ how to do this, adapting the notion of stringy mixed Hodge numbers as they appear e.g. in Batyrev-Dais [6]. These in turn are hybrids of the stringy Hodge numbers of Vafa [42] and Zaslow [44] with the mixed Hodge numbers of Deligne [12, 13].

Perhaps this is the moment to confess that the relationship between the Hodge numbers of our mirror partners is not the usual one. The familiar identity between Hodge numbers of mirror partners $M$ and $\hat{M}$ is of the form $h^{p, q}(\hat{M})=h^{\operatorname{dim} M-p, q}(M)$. We will see, however, that our mirror partners satisfy an identity of a simpler form: just $h^{p, q}(\hat{M})=h^{p, q}(M)$. This seems to reflect the fact that they are hyperkähler and noncompact. At any rate, compact hyperkähler manifolds (and orbifolds) satisfy $h^{p, q}(M)=h^{\operatorname{dim} M-p, q}(M)$, and hence we expect $h^{p, q}(\hat{M})=h^{p, q}(M)$ for compact hyperkähler mirror partners. Apparently this relationship persists in the noncompact case, even though the familiar mirror identity does not.

A physical explanation of this based on the original quantum field theory would be gratifying. But we must also bear in mind that, for noncompact varieties, the mixed Hodge numbers, and hence our Hodge numbers, depend on the algebraic structure. (Indeed, the spaces of representations of the fundamental group — what Simpson [35] calls the Betti spaces - are analytically but not algebraically isomorphic to our spaces, and their Hodge numbers will in general be different.) This seems hard to explain from a physical point of view. It might be preferable to work with some notion of Hodge numbers depending on the metric and not the algebraic structure.

Nevertheless, the equality of Hodge numbers that we uncover is striking and totally 
unexpected from a mathematical viewpoint. At any rate, it follows from the equality of terms contributed by loci in the moduli space which seem to be completely unrelated to one another. They are fixed loci of natural group actions, but on one side, the group acting is $\mathbb{C}^{\times}$, while on the other it is a finite abelian group $\Gamma$. So our result illustrates both the power and the mystery of mirror symmetry.

Here is a sharper outline of the contents of the paper. The first two sections review the known facts we will need: covers Higgs bundles, flat connections, and the Hitchin system. The next section is devoted to the proof of our first main result, Theorem (3.7), showing that the moduli spaces of flat connections on a curve with structure groups $\mathrm{SL}_{r}$ and $\mathrm{PGL}_{r}$ are $\mathrm{SYZ}$ mirror partners.

The rest of the paper is devoted to the evaluation of Hodge numbers for these spaces. In $\S$ 团 we define the appropriate notion of Hodge numbers: stringy mixed Hodge numbers with coefficient system provided by a flat unitary gerbe! This enables us to state our main conjecture, Conjecture (5.1), on the equality of the Hodge numbers for the $\mathrm{SL}_{r}$ and $\mathrm{PGL}_{r}$ spaces, which we then proceed to prove for $r=2$ and 3 .

It is much easier to work with Higgs bundles than flat connections, because of the algebraic $\mathbb{C}^{\times}$-action on the moduli space. So we begin our proof by showing in $\$ 6$ that these two moduli spaces have the same Hodge numbers, and thereafter we work exclusively with the space of Higgs bundles. In $\$ 7$ we describe (following Narasimhan-Ramanan [30]) the fixed points of the action on the $\mathrm{SL}_{r}$ moduli space of the group $\Gamma$ of $r$-torsion points in the Jacobian, and in $\$ 8$ we use this to compute the Hodge numbers of the $\mathrm{PGL}_{r}$ space. Then in $\$ 9$ we describe (following Hitchin [24 and Gothen [17]) the fixed points of the action of $\mathbb{C}^{\times}$, and in $\$ 10$ we use this to compute the Hodge numbers of the $\mathrm{SL}_{r}$ space - in sufficient detail that, for $r=2$ and 3, we get a complete answer.

The main results of this paper were announced in a note in 2001 [22]. The Proposition and Theorem 3 in that announcement correspond roughly to Theorems (3.7) and (10.6) in the present work. But the latter results actually represent substantial improvements: in particular, the meaning and function of the $B$-field have been greatly clarified. For example, Theorem 3 of the announcement refers to stringy Hodge numbers with discrete torsion; although the numbers turn out to be the same, we now understand that the canonical $B$ field, as defined in $\S 3$, is a flat gerbe which may not come from discrete torsion. Theorems 1 and 2 of the announcement concern the spaces of flat connections on punctured curves, or equivalently, of parabolic Higgs bundles; once the $B$-field is properly understood, this is mostly parallel to the present case, and we intend to treat it elsewhere.

One word about terminology: we use torsors liberally in the paper, both for sheaves of groups and for group schemes, so here is a definition. A torsor for a sheaf of groups $T$ over a base $X$ is a sheaf of $T$-spaces over $X$ which is locally isomorphic to $T$ as a sheaf with $T$-action. The same definition holds if sheaves are replaced by schemes, or even by families in a $C^{\infty}$ sense. If $X$ is a point, then a $T$-torsor is a principal homogeneous space for $T$ : it can be identified with $T$ up to the choice of a basepoint. In this paper, the relevant groups are always abelian. 
Acknowledgements. We are very grateful to Nigel Hitchin, who suggested the germ of the idea for this work as long ago as 1996; and to Pierre Deligne, Ron Donagi, Dennis Gaitsgory, Tony Pantev, and Balázs Szendrői for helpful remarks. We also thank Cumrun Vafa for drawing our attention to an earlier paper [7] treating related ideas in a physical context.

\section{Strominger-Yau-Zaslow}

Calabi-Yau manifolds and B-fields. We take a Calabi-Yau manifold to be a complex manifold equipped with a Ricci-flat Kähler metric. On a Calabi-Yau manifold of complex dimension $n$, parallel transport defines on any simply connected neighborhood a covariant constant holomorphic $n$-form $\Omega$, unique up to a scalar. Usually — as for example when $M$ itself is simply connected — this form is defined globally, and we assume this for simplicity.

Mirror symmetry is supposed to relate two such Calabi-Yau manifolds $M$ and $\hat{M}$, interchanging the deformation spaces of the Kähler and complex structures. However, the Kähler forms are real 2-forms of type $(1,1)$; to allow the Kähler deformations to be complex, we choose auxiliary fields, say $B$ on $M$ and $\hat{B}$ on $\hat{M}$, which are in some sense imaginary parts for the Kähler forms. Exactly where the $B$-field takes values is not entirely clear in the physics literature, but following a suggestion of Hitchin [26] we will take it to be an element of $H^{2}(M, \mathrm{U}(1))$, or an isomorphism class of flat unitary gerbes. By this we mean the following.

Gerbes and their trivializations. Let $T$ be a sheaf of abelian groups over a variety $M$ (with the complex or étale topology). A Picard category is a tensor category where all objects and all morphisms are invertible. The category of $T$-torsors constitutes a sheaf of Picard categories (or stack) over $M$. Sheaf of categories here means roughly what one would expect, but the precise definition is somewhat technical; a convenient reference is Donagi-Gaitsgory [14].

A T-gerbe is a sheaf of categories which is a torsor over this sheaf. That is, the sheaf consisting of $T$-torsors must act on the gerbe, and be locally equivalent to it as a sheaf with this action. For us, $T$ will usually be the sheaf of locally constant functions with values in $\mathrm{U}(1)$; then $\mathrm{U}(1)$-torsors are flat unitary line bundles, and we refer to U(1)-gerbes as flat unitary gerbes.

An isomorphism of $T$-gerbes is an equivalence of sheaves of categories as torsors over the sheaf of $T$-torsors. An automorphism is a self-isomorphism; since a gerbe is a torsor over the sheaf of $T$-torsors, its automorphisms are identified with sections of that sheaf, that is, with $T$-torsors, acting by tensorization. A trivialization of a $T$-gerbe is an isomorphism to the trivial gerbe. Two trivializations $z, z^{\prime}$ are equivalent if the automorphism $z^{\prime} \circ z^{-1}$ is given by tensorization with a trivial $T$-torsor. The space of equivalence classes of trivializations of a (trivial) gerbe $B$ will be denoted $\operatorname{Triv}^{T}(M, B)$; it is naturally an $H^{1}(M, T)$-torsor over a point.

The key result on gerbes is due to Giraud [9, 16]: it asserts that isomorphism classes of $T$ - 
gerbes are in one-to-one correspondence with $H^{2}(M, T)$. Indeed, to construct a Čech cocycle $x \in C^{2}(M, T)$ from a gerbe, choose a cover $\left\{U_{\alpha}\right\}$ such that the gerbe is trivialized on each $U_{\alpha}$. The overlaps are then given by tensorizations by $T$-torsors $L_{\alpha, \beta}$, with $L_{\alpha, \beta} \otimes L_{\beta, \gamma} \otimes L_{\gamma, \alpha}$ canonically trivialized on the triple overlaps. After refining the cover if necessary, trivialize each $L_{\alpha, \beta}$, and then compare with the canonical trivializations on the triple overlaps to get the cocycle. In this setting, a trivialization can be regarded as a cochain $y \in C^{1}(M, T)$ with $d y=x$, and two trivializations are equivalent if they differ by an exact cocycle. Then it is clear why equivalence classes of trivializations form an $H^{1}(M, T)$-torsor.

Orbifolds. Strictly speaking, the mirror of a Calabi-Yau manifold may not be a manifold, but rather an orbifold. The notion of a Calabi-Yau orbifold is defined in Appendix A of Cox-Katz [10], and on such orbifolds, gerbes may be defined much as line bundles are.

For the present purposes no difficult theory is needed, as the orbifolds we encounter are all global quotients of Calabi-Yau manifolds by the actions of finite groups. If $M=X / \Gamma$ is a quotient of this kind, and $T$ is a sheaf over $X$ to which the action of $\Gamma$ lifts, then a $T$-gerbe on $M$ is simply a $T$-gerbe on $X$ equipped with a lifting of the $\Gamma$-action.

For example, if $X$ is a point, then a $\Gamma$-equivariant $\mathrm{U}(1)$-gerbe is a homomorphism from $\Gamma$ to the category of $\mathrm{U}(1)$-torsors over a point, which is nothing but a central extension of $\Gamma$ by $U(1)$. Such extensions are classified up to isomorphism by the group cohomology $H^{2}(\Gamma, \mathrm{U}(1))$. In the physics literature, this last group is called the discrete torsion of $\Gamma$ 43; in the mathematics literature, it is called the Schur multiplier [27].

Strominger-Yau-Zaslow. With all this understood, the proposal of Strominger-YauZaslow can be described as follows.

A torus $L$ of real dimension $n$ embedded in a Calabi-Yau $n$-orbifold is said to be special Lagrangian if $\left.\omega\right|_{L}=0$ and $\left.\operatorname{Im} \Omega\right|_{L}=0$.

Two Calabi-Yau $n$-orbifolds $M$ and $\hat{M}$, equipped with flat unitary gerbes $B$ and $\hat{B}$, respectively, are said to be $S Y Z$ mirror partners if there exist an orbifold $N$ of real dimension $n$ and smooth surjections $\mu: M \rightarrow N$ and $\hat{\mu}: \hat{M} \rightarrow N$ such that for every $x \in N$ which is a regular value of $\mu$ and $\hat{\mu}$, the fibers $L_{x}=\mu^{-1}(x) \subset M$ and $\hat{L}_{x}=\hat{\mu}^{-1}(x) \subset \hat{M}$ are special Lagrangian tori which are dual to each other in the sense that there are smooth identifications

$$
L_{x}=\operatorname{Triv}^{\mathrm{U}(1)}\left(\hat{L}_{x}, \hat{B}\right)
$$

and

$$
\hat{L}_{x}=\operatorname{Triv}^{\mathrm{U}(1)}\left(L_{x}, B\right)
$$

depending smoothly on $x$. The requirement that the identifications only be smooth is rather weak, but it is unclear what a stronger condition ought to be. Certainly isometry is too strong.

The hyperkähler case. Constructing special Lagrangian tori is usually very difficult. But suppose that $M$ is a hyperkähler manifold: that is, it has a metric which is simultaneously Kähler with respect to three complex structures $J_{1}, J_{2}, J_{3}$ satisfying the commutation relations of the quaternions $i, j, k$. Let $\omega_{1}, \omega_{2}, \omega_{3}$ be the corresponding Kähler forms. Then 
$\omega_{2}+i \omega_{3}$ is a complex symplectic form on $M$ which is holomorphic with respect to $J_{1}$. The associated volume form gives a covariant constant trivialization of the canonical bundle, which shows that $M$ is Ricci-flat and hence Calabi-Yau with respect to $J_{1}$, and by permuting the indices, with respect to all three complex structures.

In this case, it is easy to see that any complex submanifold $L \subset M$ which is complex Lagrangian with respect to $J_{1}$ is special Lagrangian with respect to $J_{2}$ [25]. So the desired family of special Lagrangian tori can be found by holomorphic methods: first find holomorphic maps $\mu$ and $\hat{\mu}$ whose generic fibers are complex Lagrangian tori, then perform a hyperkähler rotation, that is, change to a different complex structure. The Hitchin system, to be described below, gives holomorphic maps of precisely this kind on a hyperkähler manifold.

\section{Higgs bundles and local systems}

Review of the basic facts. Let us recall some of the theory of Higgs bundles and local systems on curves, as developed by Hitchin [24] and Simpson [35].

Let $C$ be a smooth complex projective curve of genus $g$. It will be convenient to fix a basepoint $c \in C$. A Higgs bundle is a pair $(E, \phi)$ consisting of a vector bundle $E$ over $C$ and a section $\phi \in H^{0}(C$, End $E \otimes K)$, where $K$ is the canonical bundle. It is stable if all proper subbundles $F \subset E$ with $\phi(F) \subset F \otimes K$ satisfy $\operatorname{deg} F / \operatorname{rk} F<\operatorname{deg} E / \operatorname{rk} E$. Hitchin and Simpson then prove the following. (The subscripts Dol, DR and Hod are Simpson's notation; they honor Dolbeault, De Rham, and Hodge respectively.)

- There exists a smooth, quasi-projective moduli space $M_{\text {Dol }}^{d}$ of stable Higgs bundles of rank $r$ and degree $d$.

- There exists a smooth, quasi-projective moduli space $M_{\mathrm{DR}}^{d}$ of irreducible local systems (that is, flat vector bundles) on $C \backslash\{c\}$ of rank $r$, with holonomy $e^{2 \pi i d / r}$ around $c$.

- These two spaces are naturally diffeomorphic; indeed, there exists an isosingular family $M_{\text {Hod }}^{d}$ over the affine line whose zero fiber is $M_{\text {Dol }}^{d}$, but whose fiber over every other closed point is $M_{\mathrm{DR}}^{d}$.

- There is a $\mathbb{C}^{\times}$-action on $M_{\text {Hod }}^{d}$ lifting the standard action on the affine line, and restricting to $M_{\text {Dol }}^{d}$ as $t \cdot(E, \phi)=(E, t \phi)$.

- There is a Riemannian metric on $M_{\text {Dol }}^{d}$ for which the Dolbeault and De Rham complex structures form part of a hyperkähler structure.

More general structure groups. If vector bundles are replaced by principal bundles, the whole theory generalizes without difficulty. Higgs bundles and local systems make sense, their moduli make sense, and even the spaces $M_{\text {Hod }}$ make sense. For example, the right notion of a principal Higgs bundle consists of a principal bundle $E$ and a section $\phi \in$ 
$H^{0}(C$, ad $E \otimes K)$. Simpson explains why there exists a moduli space of principal Higgs bundles, stable in the appropriate sense. However, for the purposes of this paper we only need structure groups $\mathrm{GL}_{r}, \mathrm{SL}_{r}$, and $\mathrm{PGL}_{r}$, so we make do with the direct descriptions of the moduli spaces below. It is easy to check that these descriptions agree with Simpson's definitions, but it is even easier to regard these descriptions as definitions themselves. In each case, we describe $M_{\mathrm{Hod}} ; M_{\mathrm{Dol}}$ and $M_{\mathrm{DR}}$ are the zero and nonzero fibers, respectively.

- Let $M_{\mathrm{Hod}}^{d}\left(\mathrm{GL}_{r}\right)=M_{\mathrm{Hod}}^{d}$ as defined above.

- Let $M_{\mathrm{Hod}}^{d}\left(\mathrm{SL}_{r}\right)$ be the inverse image of a smooth algebraic section $s \subset M_{\mathrm{Hod}}^{d}\left(\mathbb{C}^{\times}\right)$ under the morphism det : $M_{\text {Hod }}^{d}\left(\mathrm{GL}_{r}\right) \rightarrow M_{\mathrm{Hod}}^{d}\left(\mathbb{C}^{\times}\right)$induced by the determinant representation of $\mathrm{GL}_{r}$. It is convenient to take $s(0)=(\mathcal{O}(d c), 0) \in M_{\text {Dol }}^{d}\left(\mathbb{C}^{\times}\right)$, where $c \in C$ is the basepoint; then $M_{\text {Dol }}^{d}\left(\mathrm{SL}_{r}\right)$ parametrizes stable Higgs bundles $(E, \phi)$ with $\Lambda^{r} E \cong \mathcal{O}(d c)$ and $\operatorname{tr} \phi=0$.

- Let $M_{\mathrm{Hod}}^{d}\left(\mathrm{PGL}_{r}\right)$ be the geometric quotient of $M_{\mathrm{Hod}}^{d}\left(\mathrm{SL}_{r}\right)$ by the group scheme $\Gamma=$ $\mathrm{Pic}^{0} C[r]$ consisting of isomorphism classes of line bundles whose $r$ th power is trivial, acting by tensorization.

In the second item, the existence of a smooth section $s$ follows, for example, from the Białynicki-Birula decomposition theorem [8, 40], taking a $\mathbb{C}^{\times}$-orbit whose closure contains $(\mathcal{O}(d c), 0)$. To see that every section $s$ gives the same space $M_{\text {Hod }}^{d}\left(\mathrm{SL}_{r}\right)$ up to isomorphism, notice that tensorization makes $M_{\text {Hod }}^{d}\left(\mathbb{C}^{\times}\right)$into a $M_{\text {Hod }}^{0}\left(\mathbb{C}^{\times}\right)$-torsor over the affine line, so any two sections differ by multiplication by a section of the family of groups $M_{\mathrm{Hod}}^{0}\left(\mathbb{C}^{\times}\right)$. Since the $r$ th power map is étale on $M_{\mathrm{Hod}}^{0}\left(\mathbb{C}^{\times}\right)$, and the base is simply connected, there exists a smooth $r$ th root of this section, which can be used to tensorize objects in $M_{\mathrm{Hod}}^{d}\left(\mathrm{GL}_{r}\right)$.

Incidentally, the use of the notation $M_{\mathrm{Hod}}^{d}\left(\mathrm{SL}_{r}\right)$ is perhaps slightly misleading, since the objects it parametrizes do not have structure group $\mathrm{SL}_{r}$ unless $d=0$ and $s$ is the trivial section.

Everything asserted before for Higgs bundles remains true in this more general setting, except that the $\mathrm{PGL}_{r}$ moduli spaces are not smooth; rather, they are hyperkähler orbifolds.

The Hitchin system. On each of the Dolbeault spaces $M_{\text {Dol }}^{d}(G)$, there exists a completely integrable Hamiltonian system, the so-called Hitchin system or Hitchin map. It is a morphism $\mu$ from $M_{\text {Dol }}^{d}(G)$ to an affine space $V_{G}$ of half the dimension. Here $V_{\mathrm{GL}_{r}}=$ $\bigoplus_{i=1}^{n} H^{0}\left(C, K^{i}\right)$ and $V_{\mathrm{SL}_{r}}=V_{\mathrm{PGL}_{r}}=\bigoplus_{i=2}^{n} H^{0}\left(C, K^{i}\right)$. The morphism is evaluated on a Higgs bundle $(E, \phi)$ by applying to $\phi$ the invariant polynomials on the Lie algebra $\mathfrak{g}$. Hitchin shows that $\mu$ is proper when $r$ and $d$ are coprime. He also shows that the fiber over a general point is complex Lagrangian and is (a torsor for) an abelian variety.

This is exactly the situation discussed at the end of $\delta$. Consequently, for any integers $d, e \in \mathbb{Z}$, the De Rham spaces $M_{\mathrm{DR}}^{d}\left(\mathrm{SL}_{r}\right)$ and $M_{\mathrm{DR}}^{e}\left(\mathrm{PGL}_{r}\right)$ carry families of special Lagrangian tori over the same base, just as the SYZ definition requires. All that remains to be verified is the statement about duality of the tori. We will establish this in the next section, but first we need to review Hitchin's description of the fibers of $\mu$ in more detail. 
An element of $V_{G}$ is given by sections $\beta_{i} \in H^{0}\left(C, K^{i}\right)$ for $i=1$ to $n$ (taking $\beta_{1}=0$ in the $\mathrm{SL}_{r}$ and $\mathrm{PGL}_{r}$ cases). The equation

$$
z^{n}+\beta_{1} z^{n-1}+\beta_{2} z^{n-2}+\cdots+\beta_{n}=0,
$$

where $z$ lies in the total space of $K$, defines a curve $\pi: \tilde{C} \rightarrow C$ inside this total space, called the spectral cover. For $\left(\beta_{i}\right)$ in the Zariski open set $U \subset V_{G}$ where $\tilde{C}$ is smooth, $\mu^{-1}\left(\beta_{i}\right)$ can be canonically identified as follows [24].

- When $G=\mathrm{GL}_{r}$, it is $\tilde{J}^{d}=\operatorname{Pic}^{d} \tilde{C}$. This can be regarded as (the fiber of) a $\tilde{J}^{0}$-torsor over $U$.

- When $G=\mathrm{SL}_{r}$, it is $P^{d}=\mathrm{Nm}^{-1}(\mathcal{O}(d c))$, the generalized Prym variety. Here Nm : $\operatorname{Pic}^{d} \tilde{C} \rightarrow \operatorname{Pic}^{d} C$ is the norm map (see e.g. Arbarello et al. [2, App. B] for a definition and basic properties). This is a $P^{0}$-torsor over $U$.

- When $G=\mathrm{PGL}_{r}$, it is $\hat{P}^{d}=P^{d} / \Gamma$, the quotient of the Prym by the action of $\Gamma$ on $M_{\text {Dol }}^{d}\left(\mathrm{SL}_{r}\right)$, which preserves it. This is a $\hat{P}^{0}$-torsor over $U$.

The next two lemmas explain how these torsors are related to one another.

(2.2) Lemma. Let $J^{0}=\operatorname{Pic}^{0} C$. Then there is a natural isomorphism

$$
\tilde{J}^{d} \cong \frac{P^{d} \times J^{0}}{\Gamma}
$$

under which $\mathrm{Nm}$ corresponds to the projection to $J^{0} / \Gamma$ followed by the isomorphism $J^{0} / \Gamma \rightarrow$ $J^{0}$ given by taking $-r$ th powers.

Proof. Certainly there is a morphism $P^{d} \times J^{0} \rightarrow \tilde{J}^{d}$ given by $(L, M) \mapsto L \otimes \pi^{*} M^{-1}$, whose composition with $\mathrm{Nm}$ is $(L, M) \mapsto M^{-r}$. This morphism is invariant under the action of $\Gamma$ by tensorization on both factors. It therefore suffices to show that $P^{0} \cap \pi^{*} J^{0}=\pi^{*} \Gamma$ and that $\pi^{*}: J^{0} \rightarrow \tilde{J}^{0}$ is injective.

Since $\operatorname{Nm} \pi^{*} L=L^{r}$, certainly ker $\pi^{*} \subset \Gamma$ and $P^{0} \cap \pi^{*} J^{0}=\operatorname{ker} \operatorname{Nm} \cap \pi^{*} J^{0}=\pi^{*} \Gamma$, which proves the first assertion.

For the second, suppose $L \in \Gamma$ has order $k$ and satisfies $\pi^{*} L \cong \mathcal{O}$. The isomorphism $L^{k} \cong \mathcal{O}$ determines a $k$-valued multisection of $L$; regard this as a cover of $C$. Then $\pi: \tilde{C} \rightarrow$ $C$ must factor through this cover: indeed, the trivialization of $\pi^{*} L$ gives a trivialization of $\pi^{*} L^{k}$, so (after multiplication by an overall constant) it lies in the pullback of, and so defines a morphism to, the multisection.

However, the only connected unbranched cover of $C$ through which $\pi$ factors is the trivial cover. This is clear when $\pi$ has a point of total ramification, which occurs when all the coefficients $\beta_{i}$ of (2.1) have a common zero. But it is also clearly invariant under continuous deformations, hence true everywhere on the connected base $U \subset V_{G}$.

Therefore $k=1$, so $\pi^{*}$ has trivial kernel. 
(2.3) Lemma. The dual of $P^{0}$ is $\hat{P}^{0}=P^{0} / \Gamma$.

Proof. Just dualize the short exact sequence

$$
0 \longrightarrow P^{0} \longrightarrow \tilde{J}^{0} \stackrel{\mathrm{Nm}}{\longrightarrow} J^{0} \longrightarrow 0
$$

to get

$$
0 \longrightarrow J^{0} \stackrel{\pi^{*}}{\longrightarrow} \tilde{J}^{0} \longrightarrow P^{0} \longrightarrow 0,
$$

where $\hat{P}^{0}=\tilde{J}^{0} / J^{0}=P^{0} / \Gamma$ by the previous lemma.

\section{Trivializations of the $B$-fields}

With the prerequisites complete, we proceed in this section to our first goal. This is to show that, when equipped with certain $B$-fields, the De Rham moduli spaces with structure groups $G$ and $\hat{G}$ are SYZ mirror partners. Our expectation is that this will hold true for any reductive $G$, but at present we confine ourselves to the case $G=\mathrm{SL}_{r}, \hat{G}=\mathrm{PGL}_{r}$.

In fact, we deduce the smooth identification of special Lagrangian tori on the De Rham spaces, called for by SYZ, from a stronger statement: a holomorphic identification of complex Lagrangian tori on the Dolbeault spaces. The two are related by hyperkähler rotation as discussed at the end of 8 . Moreover, since the smooth parts of these two spaces are diffeomorphic, flat unitary gerbes on them can be identified. Therefore, in this section, we work exclusively with the Dolbeault space and, for brevity, denote the stable part of $M_{\mathrm{Dol}}^{d}\left(\mathrm{SL}_{r}\right)$ simply by $M_{\mathrm{Dol}}^{d}$.

We will work over $U$, the Zariski open set in the range of the Hitchin map $\mu$ where the spectral cover $\tilde{C}$ is smooth. The four torsors over $U$ that concern us are $\tilde{J}^{d}, P^{d}$, and $\hat{P}^{d}$, as defined in $\oint$, plus $J^{d}=\operatorname{Pic}^{d} C$, which we regard as a trivial $J^{0}$-torsor over $U$.

Any of the methods used to construct universal families on the moduli space of ordinary stable bundles adapt without change to the space of Higgs bundles $M_{\text {Dol }}^{d}$. For example, one could use descent and the geometric invariant theory construction of $M_{\text {Dol }}^{d}$ given by Nitsure [32]. Provided that $r$ and $d$ are coprime, one gets a bona fide universal Higgs pair $(\mathbf{E}, \boldsymbol{\Phi}) \rightarrow M_{\text {Dol }}^{d} \times C$. However, as for stable bundles, the scalars, acting as automorphisms, provide an obstruction to the existence of $\mathbf{E}$ when $r$ and $d$ are not coprime. The best we can do in general is to construct a universal projective bundle and a universal endomorphism bundle, abusively denoted $\mathbb{P E}$ and End $\mathbf{E}$ even though $\mathbf{E}$ does not exist, and a universal Higgs field $\mathbf{\Phi} \in H^{0}($ End $\mathbf{E} \otimes K)$. Then the restriction $\left.\mathbb{P} \mathbf{E}\right|_{M_{\text {Dol }}^{d} \times\{c\}}$ to the basepoint in $C$ is a projective bundle $\Psi$ on $M_{\text {Dol }}^{d}$.

Let $B$ be the gerbe of liftings of $\Psi$, meaning the sheaf of categories on $M$ taking an étale neighborhood to the category of liftings on that neighborhood of $\Psi$ to an $\mathrm{SL}_{r}$-bundle. Since any two liftings differ by tensorization by a $\mathbb{Z}_{r}$-torsor, $B$ is a $\mathbb{Z}_{r}$-gerbe. 
(3.1) Lemma. The restriction of $B$ to each regular fiber $P^{d}$ of the Hitchin map is trivial as a $\mathbb{Z}_{r}$-gerbe.

Proof. It suffices to show that $\left.\Psi\right|_{P^{d}}$ does in fact lift to an $\mathrm{SL}_{r}$-bundle, which can be regarded as a vector bundle with trivial determinant.

For any universal bundle $\tilde{\mathcal{L}} \rightarrow P^{d} \times \tilde{C}$, the push-forward $\pi_{*} \tilde{\mathcal{L}} \rightarrow P^{d} \times \tilde{C}$ admits a family of Higgs fields inducing the inclusion $P^{d} \subset M$. Indeed, this is how one shows that $P^{d}$ is the fiber of the Hitchin map: see Hitchin [24] for details. So over $P^{d} \times\{c\}$ there is an isomorphism $\mathbb{P} \pi_{*} \tilde{\mathcal{L}} \cong \Psi$.

The universal bundle can be normalized so that $\left.\tilde{\mathcal{L}}\right|_{P^{d} \times\{y\}} \in \operatorname{Pic}^{0}\left(P^{d}\right)$ for one (hence all) $y \in \tilde{C}$. The determinant of $\pi_{*} \tilde{\mathcal{L}}$ over $P^{d} \times\{c\}$ is isomorphic to $\left.\bigotimes_{y \in \pi^{-1}(c)} \tilde{\mathcal{L}}\right|_{P^{d} \times\{y\}}$, where ramification points are counted with the appropriate multiplicity. This has an $r$ th root, tensoring by whose inverse will further adjust the normalization of $\tilde{\mathcal{L}}$ so that $\left.\operatorname{det} \pi_{*} \tilde{\mathcal{L}}\right|_{P^{d} \times\{c\}} \cong$ $\mathcal{O}$, making $\left.\pi_{*} \tilde{\mathcal{L}}\right|_{P^{d} \times\{c\}}$ an $\mathrm{SL}_{r}$-bundle as desired.

Now that we know that $B$ restricts trivially to each fiber as a $\mathbb{Z}_{r}$-gerbe, and hence as a $\mathrm{U}(1)$-gerbe, it makes sense to examine the equivalence classes of $\mathrm{U}(1)$-trivializations. From the discussion of gerbes in $\delta$, we know that these form a torsor (in the smooth category) for $H^{1}\left(P^{d}, \mathrm{U}(1)\right) \cong \mathrm{Pic}^{0} P^{d} \cong \mathrm{Pic}^{0} P^{0}$, and from Lemma (2.3) the latter is $\hat{P}^{0}$.

(3.2) Proposition. For any $d, e \in \mathbb{Z}$, there is a smooth isomorphism of $\hat{P}^{0}$-torsors

$$
\operatorname{Triv}^{\mathrm{U}(1)}\left(P^{d}, B^{e}\right) \cong \hat{P}^{e} .
$$

Proof. The isomorphism classes of torsors over a fixed abelian group scheme themselves form an abelian group in a natural way, and it is easy to see that $\hat{P}^{e} \cong\left(\hat{P}^{1}\right)^{e}$ and $\operatorname{Triv}\left(P, B^{e}\right) \cong$ $(\operatorname{Triv}(P, B))^{e}$, where the right-hand sides are $e$ th powers under this group operation. Hence it suffices to take $e=1$.

As seen in the proof of Lemma (3.1), the triviality of $B$ on $P^{d}$ follows from the existence of a universal bundle $\tilde{\mathcal{L}} \rightarrow P^{d} \times \tilde{C}$ with $\operatorname{det} \pi_{*} \tilde{\mathcal{L}}$ trivial on $P^{d} \times\{c\}$. Consider the set of isomorphism classes of all such $\tilde{\mathcal{L}}$ : this parametrizes the equivalence classes of trivializations of $B$ as a $\mathbb{Z}_{r}$-gerbe, which is to say, it forms the torsor $\operatorname{Triv}^{\mathbb{Z}_{r}}\left(P^{d}, B\right)$. It is a $\hat{P}^{0}[r]$-torsor over $U$, where $\hat{P}^{0}[r]=H^{1}\left(P^{d}, \mathbb{Z}_{r}\right)$ are the torsion points of order $r$ in $\hat{P}^{0}$. This makes sense, since for $L \in \hat{P}^{0}=\operatorname{Pic}^{0}\left(P^{d}\right)$, the push-pull formula says

$$
\operatorname{det} \pi_{*}\left(\pi^{*} L \otimes \tilde{\mathcal{L}}\right)=L^{r} \otimes \operatorname{det} \pi_{*} \tilde{\mathcal{L}} .
$$

We are really interested not only in $\mathbb{Z}_{r}$-trivializations but in all $\mathrm{U}(1)$-trivializations. These comprise a torsor for $\hat{P}^{0}=H^{1}\left(P^{d}, \mathrm{U}(1)\right)$ containing the $\hat{P}^{0}[r]$-torsor above, and indeed this property determines the larger torsor, since it can be identified with the quotient

$$
\frac{\operatorname{Triv}^{\mathbb{Z}_{r}}\left(P^{d}, B\right) \times \hat{P}^{0}}{\hat{P}^{0}[r]}
$$


An obvious torsor with this property consists of all universal bundles $\tilde{\mathcal{L}} \rightarrow P^{d} \times \tilde{C}$ with $\left.\tilde{\mathcal{L}}\right|_{P^{d} \times\{y\}} \in \operatorname{Pic}^{0}\left(P^{d}\right)$ for any $y \in \tilde{C}$. It therefore suffices to show that this torsor is isomorphic to $\hat{P}^{1}$.

In fact, $\hat{P}^{1}=\tilde{J}^{1} / J^{0}$, while the torsor of the previous paragraph is also a quotient by $J^{0}$, of the torsor consisting of universal bundles as stated there, except with $P^{d}$ replaced by $\tilde{J}^{d}$. (The $J^{0}$-action comes from tensoring by $\pi^{*}$ Pic $J^{0} \cong J^{0}$.) So it actually suffices to show that the latter torsor is isomorphic to $\tilde{J}^{1}$ as a $\tilde{J}^{0}$-torsor. To do this, we will exhibit morphisms $f_{1}$ and $f_{2}$ from $\tilde{C}$ to the two torsors such that, for any $y, y^{\prime} \in \tilde{C}$, $f_{1}\left(y^{\prime}\right)-f_{1}(y)=f_{2}\left(y^{\prime}\right)-f_{2}(y) \in \hat{P}^{0}$. The isomorphism of the two torsors defined by identifying $f_{1}(y)$ with $f_{2}(y)$ is then independent of $y$, and hence well-defined.

The morphism $f_{1}$ is simply the Abel-Jacobi map $\tilde{C} \rightarrow \tilde{J}^{1}$. As for $f_{2}$, it takes $y$ to the universal bundle whose restriction to $P^{d} \times\{y\}$ is trivial. The equality $f_{1}\left(y^{\prime}\right)-f_{1}(y)=$ $f_{2}\left(y^{\prime}\right)-f_{2}(y)$ then means that the restriction to $y$ of the universal bundle normalized at $y^{\prime}$ is the line bundle on $\tilde{J}^{d}$ corresponding to $f_{1}\left(y^{\prime}\right)-f_{1}(y) \in \tilde{J}^{0}=\operatorname{Pic}^{0} \tilde{J}^{d}$. This follows readily from two well-known facts. First, that this universal bundle is of the form $p_{2}^{*} L_{0} \otimes F^{*} \mathcal{P}$, where $p_{2}$ is projection on the second factor, $L_{0} \in \tilde{J}^{d}$ is fixed, $\mathcal{P}$ is the Poincaré line bundle, and

$$
F: \tilde{J}^{d} \times \tilde{C} \rightarrow \tilde{J}^{0} \times \tilde{J}^{0}
$$

is given by $F(L, y)=\left(L \otimes L_{0}^{-1}, f_{1}(y)-f_{1}\left(y^{\prime}\right)\right)$. Second, that the involution of $\tilde{J}^{0} \times \tilde{J}^{0}$ exchanging the two factors takes the Poincaré bundle to its inverse.

Now turn to the reverse direction. We need a gerbe $\hat{B}$ on the orbifold $\hat{M}_{\text {Dol }}^{d}=M_{\text {Dol }}^{d} / \Gamma$, or equivalently, a $\Gamma$-equivariant gerbe on $M_{\text {Dol }}^{d}$. This will just be $B$ equipped with a $\Gamma$-action, which we define as follows. Let $L_{\gamma}$ denote the line bundle over $C$ corresponding to $\gamma \in \Gamma$. (Here and throughout, it will prove convenient to distinguish between the abstract group element $\gamma$ and the line bundle $L_{\gamma}$.) Then $\gamma$ acts on $M_{\text {Dol }}^{d}$ by $(E, \phi) \mapsto\left(E \otimes L_{\gamma}, \phi\right)$. This lifts to $\mathbb{P E}$ : think of $\mathbb{P E}$ as the moduli space parametrizing 1-dimensional subspaces of a stable Higgs bundle, and observe that tensoring by $L_{\gamma}$ induces a natural transformation. Hence $\Gamma$ acts on $\mathbb{P} \mathbf{E}$ and on its restriction $\Psi$ to $M_{\text {Dol }}^{d} \times\{c\}$. This determines a $\Gamma$-action on $B$, the sheaf of liftings to $\mathrm{SL}_{r}$-bundles, making it an equivariant flat gerbe $\hat{B}$.

To prove an analogue of Lemma (3.1) for $\hat{B}$, we first need a technical fact. Let

$$
\tilde{\Gamma}=\bigsqcup_{\gamma \in \Gamma} L_{\gamma} \backslash 0
$$

be the disjoint union of the total spaces of the line bundles $L_{\gamma}$, minus their zero sections.

(3.3) Lemma. This has the structure of a group scheme over $C$ whose fiber at $y \in C$ is an abelian extension

$$
1 \longrightarrow \mathbb{C}^{\times} \longrightarrow \tilde{\Gamma}_{y} \longrightarrow \Gamma \longrightarrow 0 .
$$

If $\mathcal{L} \rightarrow J^{0} \times C$ is the universal bundle which is trivial on $J^{0} \times\{c\}$, then there is an action over $C$ of $\tilde{\Gamma}$ on the total space of $\mathcal{L}$, lifting the action of $\Gamma$ on $J^{0}$ by translation, so that the scalars $\mathbb{C}^{\times}$act with weight 1 on the fibers. 
Of course the above extension always splits, but not canonically except at the basepoint $y=c$.

Proof. Let $A$ be an abelian variety (which we will shortly take to be $J^{0}$ ), let $\hat{A}$ be its dual, and let $\mathcal{P} \rightarrow A \times \hat{A}$ be the Poincaré bundle trivialized on $(0 \times \hat{A}) \cup(A \times \hat{0})$, where $0 \in A$, $\hat{0} \in \hat{A}$ are the basepoints. It is well-known, cf. Serre [34, VII 3.16], that $\operatorname{Ext}^{1}\left(A, \mathbb{C}^{\times}\right)=\hat{A}$; indeed, $\hat{A}$ parametrizes a family of abelian central extensions of $A$ by $\mathbb{C}^{\times}$. If this is regarded as a group scheme over $\hat{A}$, then its total space is $\mathcal{P} \backslash 0$, the complement of the zero section in $\mathcal{P}$. The group operation over $\hat{A}$ is given by an isomorphism over $A \times A \times \hat{A}$

$$
p_{13}^{*} \mathcal{P} \otimes p_{23}^{*} \mathcal{P} \cong(m \times 1)^{*} \mathcal{P},
$$

where $p_{13}$ and $p_{23}$ are projections on the relevant factors and $m: A \times A \rightarrow A$ is addition. This is provided by the theorem of the cube [28]. It can be chosen so that over $0 \times \hat{0}$ it is 1 . Associativity requires the commutativity of a certain diagram of isomorphisms of line bundles on $A \times A \times A \times \hat{A}$, but this is automatic since the base is projective and connected, and the desired commutativity holds automatically over the base points.

In the same way, the action of the group scheme $A \times \hat{A} \rightarrow \hat{A}$ on itself by translating the first factor lifts to an action of $\mathcal{P} \backslash 0 \rightarrow \hat{A}$ on the Poincaré bundle $\mathcal{P} \rightarrow A \times \hat{A} \rightarrow \hat{A}$. Indeed, the action $(\mathcal{P} \backslash 0) \times{ }_{\hat{A}} \mathcal{P} \rightarrow \mathcal{P}$ is again given by the isomorphism (3.4). The condition that an action must satisfy is automatic for the same reason as before.

Now return to our curve $C$ with basepoint $c$, and use the Abel-Jacobi map to embed it in its Jacobian $J^{0}$ so that $c$ maps to the basepoint 0 . Let $\tilde{\Gamma}$ be the inverse image of $\Gamma \times C$ in the projection $\mathcal{P} \backslash 0 \rightarrow J^{0} \times J^{0}$. Then $\tilde{\Gamma}$ clearly satisfies the desired properties. The restriction of the Poincaré bundle on $J^{0} \times J^{0}$ to $J^{0} \times C$ is the universal bundle $\mathcal{L}$; this therefore carries the desired action.

(3.5) Lemma. The restriction of $\hat{B}$ to each regular fiber $\hat{P}^{d}$ of the Hitchin map is trivial as a $\mathbb{Z}_{r}$-gerbe.

Proof. First of all, rather than working on the orbifold $\hat{M}_{\text {Dol }}^{d}$ and restricting to $\hat{P}^{d}$, it is equivalent to work with $\Gamma$-equivariant objects on $M_{\text {Dol }}^{d}$ and restrict to $P^{d}$.

To show that $\left.\hat{B}\right|_{P^{d}}$ is trivial, it suffices to show that the projective bundle $\left.\Psi\right|_{P^{d}}$ lifts to a $\Gamma$-equivariant vector bundle with trivial determinant. We know from Lemma (3.1) that $\left.\Psi\right|_{P^{d}}$ is the projectivization of a vector bundle, but we need to show that $\Gamma$ acts on this vector bundle.

Take a universal bundle over $\tilde{J}^{d} \times \tilde{C}$; since by Lemma (2.2)

$$
\tilde{J}^{d} \cong \frac{P^{d} \times J^{0}}{\Gamma}
$$

the pullback of this bundle to $P^{d} \times J^{0} \times \tilde{C}$ has a natural $\Gamma$-action, which of course can be regarded as a $\tilde{\Gamma}$-action where the scalars $\mathbb{C}^{\times}$act trivially. By the theorem of the cube 28 this pullback is equivariantly isomorphic to $p_{13}^{*} \tilde{\mathcal{L}} \otimes p_{23}^{*}(1 \times \pi)^{*} \mathcal{L}^{-1}$, where $p_{13}$ and $p_{23}$ are 
projections on the relevant factors, and $\pi: \tilde{C} \rightarrow C$ is the spectral cover. By Lemma (3.3), $\tilde{\Gamma}$ acts on the second factor in this tensor product, with the scalars acting with weight -1 . Hence it also acts on the first, with the scalars acting with weight 1 . Restricting to the basepoint in $J^{0}$ gives us a $\tilde{\Gamma}$-action on $\tilde{\mathcal{L}} \rightarrow P^{d} \times \tilde{C}$, and hence on $\pi_{*} \tilde{\mathcal{L}} \rightarrow P^{d} \times C$. Since $\tilde{\Gamma}_{c} \cong \Gamma \times \mathbb{C}^{\times}$as mentioned before, this produces a $\Gamma$-action on $\left.\pi_{*} \tilde{\mathcal{L}}\right|_{P^{d} \times\{c\}}$.

Finally, as in the proof of Lemma (3.1), observe that $\left.\operatorname{det} \pi_{*} \tilde{\mathcal{L}}\right|_{P^{d} \times\{c\}} \in \operatorname{Pic}_{\Gamma}^{0} P^{d}=\operatorname{Pic}^{0} \hat{P}^{d}$. So, by tensoring by an equivariant line bundle, the determinant may be made equivariantly trivial.

Again we may examine the equivalence classes of $\mathrm{U}(1)$-trivializations, which now form a torsor for $H^{1}\left(\hat{P}^{d}, \mathrm{U}(1)\right) \cong P^{0}$.

(3.6) Proposition. For any $d, e \in \mathbb{Z}$, there is a smooth isomorphism of $P^{0}$-torsors

$$
\operatorname{Triv}^{\mathrm{U}(1)}\left(\hat{P}^{d}, \hat{B}^{e}\right) \cong P^{e} \text {. }
$$

Proof. First, it suffices as in the proof of Proposition (3.2) to take $e=1$.

Second, rather than working on $\hat{M}_{\text {Dol }}^{d}$, it is equivalent to work $\Gamma$-equivariantly on $M_{\text {Dol }}^{d}$. For example, the torsor $\operatorname{Triv}^{\mathbb{Z}_{r}}\left(\hat{P}^{e}, \hat{B}\right)$ parametrizing trivializations over $\hat{P}^{d}$ can be identified with the torsor $\operatorname{Triv}_{\Gamma}^{\mathbb{Z}_{r}}\left(P^{e}, B\right)$ parametrizing $\Gamma$-equivariant trivializations over $P^{d}$. As seen in the proof of the previous lemma, such trivializations are provided by $\tilde{\Gamma}$-equivariant universal bundles $\tilde{\mathcal{L}} \rightarrow P^{d} \times \tilde{C}$ where the scalars $\mathbb{C}^{\times} \subset \tilde{\Gamma}$ act with weight 1 and $\left.\operatorname{det} \pi_{*} \tilde{\mathcal{L}}\right|_{P^{d} \times\{c\}}$ is trivial. Indeed, the isomorphism classes of such universal bundles form a torsor for $\operatorname{Pic}_{\Gamma}^{0} P^{d}[r]=P^{0}[r]$, which must be precisely $\operatorname{Triv}_{\Gamma}^{\mathbb{Z}_{r}}\left(P^{e}, B\right)$.

Now follow the proof of Proposition (3.2): let $\mathcal{T}$ be the torsor for $P^{0}=\operatorname{Pic}_{\Gamma}^{0} P^{d}$ parametrizing bundles $\tilde{\mathcal{L}}$ that satisfy all the conditions of the previous paragraph save that $\left.\operatorname{det} \pi_{*} \tilde{\mathcal{L}}\right|_{P^{d} \times\{c\}}$ need only lie in $\operatorname{Pic}_{\Gamma}^{0} P^{d}$. This contains the aforementioned $P^{0}[r]$-torsor and hence must be isomorphic to $\operatorname{Triv}^{\mathrm{U}(1)}\left(\hat{P}^{e}, \hat{B}\right)$.

It remains only to identify $\mathcal{T}$ with $P^{1}$. First, recall that the $\tilde{J}^{0}$-torsor of all universal bundles $\tilde{\mathcal{L}} \rightarrow \tilde{J}^{d} \times \tilde{C}$ with $\left.\tilde{\mathcal{L}}\right|_{\tilde{J}^{d} \times\{c\}} \in \operatorname{Pic}^{0} \tilde{J}^{d}$ is isomorphic to $\tilde{J}^{1}$, as shown in the proof of Proposition (3.2).

Then notice that there is an inclusion of $\mathcal{T}$ into this torsor compatible with the inclusion $P^{0} \subset \tilde{J}^{0}$. It is given simply by tensoring $\tilde{\mathcal{L}} \rightarrow P^{d} \times \tilde{C}$ by the fixed $\tilde{\Gamma}$-equivariant bundle $\pi^{*} \mathcal{L}^{-1} \rightarrow J^{0} \times \tilde{C}, \mathcal{L}$ being the universal bundle on $J^{0} \times C$, to get a $\Gamma$-equivariant bundle over $P^{d} \times J^{0} \times \tilde{C}$, which descends to a universal bundle on the quotient $\tilde{J}^{d} \times \tilde{C}$.

So $\mathcal{T}$ and $P^{1}$ are now both $P^{0}$-subtorsors of $\tilde{J}^{1}$. The quotient by either is the constant torsor $J^{0}$. The image of one in the quotient by the other therefore gives a morphism from the base $U$ to $J^{0}$, the Jacobian of $C$. But $U$ is a Zariski open set in an affine space, so its only morphisms to an abelian variety are constants. Indeed, any nonconstant morphism would be nonconstant on some line nontrivially intersecting $U$; the closure of the image of this line would then be a rational curve in $J^{0}$, which doesn't exist.

Hence $\mathcal{T}$ and $P^{1}$ are translates of one another in $\tilde{J}^{1}$, so they are isomorphic. 
We may summarize the results of this section as follows.

(3.7) Theorem. For any $d, e \in \mathbb{Z}$, the moduli spaces $M_{\mathrm{DR}}^{d}\left(\mathrm{SL}_{r}\right)$ and $M_{\mathrm{DR}}^{e}\left(\mathrm{PGL}_{r}\right)$, equipped with the flat unitary orbifold gerbes $B^{e}$ and $\hat{B}^{d}$ respectively, are $S Y Z$ mirror partners.

\section{Stringy mixed Hodge numbers}

Since the spaces we study are non-compact and singular, their "Hodge numbers" must be interpreted in a generalized sense: as stringy mixed Hodge numbers. Mixed Hodge numbers are alternating sums of dimensions of the associated graded spaces in Deligne's mixed Hodge structures on cohomology. They are defined for any complex algebraic variety, even incomplete or singular ones. However, for the varieties with orbifold (or more generally, Gorenstein) singularities arising in string theory, mixed Hodge numbers are not the appropriate notion: rather, we need a stringy version to take proper account of the singularities. For complete smooth varieties, these stringy mixed Hodge numbers will coincide with the ordinary Hodge numbers. More generally, they coincide with the Hodge numbers of a crepant resolution, if this exists [11].

It is convenient to encode the mixed Hodge numbers as coefficients of a polynomial: the so-called E-polynomial, or virtual Hodge polynomial. We will define a stringy E-polynomial in terms of the ordinary one.

The stringy $\boldsymbol{E}$-polynomial. The stringy $E$-polynomial is defined for any Gorenstein variety, but it is expressed by a particularly simple formula in the case of a quotient $M / \Gamma$, where $M$ is a quasi-projective Calabi-Yau $n$-manifold on which the finite group $\Gamma$ acts preserving the holomorphic $n$-form $\Omega$. We will treat this formula as a definition, and present a generalization for $M$ equipped with a flat unitary orbifold gerbe $B$. The problems of how to interpret this generalization in terms of smoothings of $M$, and how to extend it to arbitrary Gorenstein varieties, are of the utmost interest, but we do not pursue them here.

To any complex variety $X$, not necessarily smooth or projective, Deligne [12, 13] has associated a canonical mixed Hodge structure on the compactly supported cohomology $H_{\text {cpt }}^{*}(X, \mathbb{C})$, and hence, passing to the associated graded, complex vector spaces $H^{p, q ; k}(X)$. These agree with $H^{p, q}(X)$ in the smooth projective case, but in general they can be nonzero even when $p+q \neq k$. If a finite group $\Gamma$ acts on $X$, it acts as well on each $H^{p, q ; k}(X)$; denote by $h^{p, q}(X)^{\Gamma}$ the alternating sum over $k$ of the dimensions of the $\Gamma$-invariant subspaces.

Then define $E(X)^{\Gamma}$ to be the polynomial in $u$ and $v$ given by

$$
E(X)^{\Gamma}=\sum_{p, q} h^{p, q}(X)^{\Gamma} u^{p} v^{q}
$$

When $\Gamma=1$, this is the virtual Hodge polynomial $E(X)$ as defined by, for example, Batyrev-Dais [6]. A practical method of determining $E(X)^{\Gamma}$, which we adopt in the proof 
of Proposition (8.2), is to regard $E(X)$ as a polynomial with coefficients in the characters of $\Gamma$, and then compute $E(X)^{\Gamma}$ as the average value over $\Gamma$.

The beauty of $E(X)^{\Gamma}$, like $E(X)$, is that it is additive for disjoint unions and multiplicative for Zariski locally trivial fibrations: the proofs given by Batyrev-Dais [6, 3.4, 3.7], for example, adapt without change to the equivariant case. This allows us to compute effectively in many cases even where we know nothing about the mixed Hodge structures.

For $M$ as above, we may now define the stringy E-polynomial to be

$$
E_{\mathrm{st}}(M / \Gamma)=\sum_{[\gamma]} E\left(M^{\gamma}\right)^{C(\gamma)}(u v)^{F(\gamma)}
$$

Here the sum runs over the conjugacy classes of $\Gamma ; C(\gamma)$ is the centralizer of $\gamma ; M^{\gamma}$ is the subvariety fixed by $\gamma$; and $F(\gamma)$ is an integer called the fermionic shift, which is defined as follows. The group element $\gamma$ has finite order, so it acts on $\left.T M\right|_{M^{\gamma}}$ as a linear automorphism with eigenvalues $e^{2 \pi i w_{1}}, \ldots, e^{2 \pi i w_{n}}$, where each $w_{j} \in[0,1)$. Let $F(\gamma)=\sum w_{j}$; this is an integer since, by hypothesis, $\gamma$ acts trivially on the canonical bundle. (Purely for convenience of notation, we have assumed that $F(\gamma)$ is the same on all components of $M^{\gamma}$; otherwise we would have to write a further sum, over these components, in the definition of $E_{\mathrm{st}}$.)

Turning on the $\boldsymbol{B}$-field. A twisted version of this expression can be formulated in the following way. Let $B$ be an orbifold $\mathrm{U}(1)$-gerbe on $M / \Gamma$, or equivalently, a $\Gamma$-equivariant $\mathrm{U}(1)$-gerbe on $M$. Such a gadget induces a flat $C(\gamma)$-equivariant line bundle $L_{B, \gamma}$ on the fixed-point set of $\gamma$. Indeed, $\left.\left.B\right|_{M^{\gamma}} \cong \gamma^{*} B\right|_{M^{\gamma}}=\left.B\right|_{M^{\gamma}}$, where the isomorphism is given by the $\Gamma$-action on $B$, and the equality is because $\gamma$ acts trivially on $M^{\gamma}$. This gives an automorphism of $B$ restricted to $M^{\gamma}$, and moreover, it is $C(\gamma)$-equivariant. Any automorphism of a $\mathrm{U}(1)$-gerbe is given by tensorization by a unique $\mathrm{U}(1)$-torsor, and this remains true equivariantly. Thus is determined a $C(\gamma)$-equivariant $\mathrm{U}(1)$-torsor on $M^{\gamma}$, which is $L_{B, \gamma}$.

We then propose the definition

$$
E_{\mathrm{st}}^{B}(M / \Gamma)=\sum_{[\gamma]} E\left(M^{\gamma} ; L_{B, \gamma}\right)^{C(\gamma)}(u v)^{F(\gamma)}
$$

where the $E$-polynomial is defined in terms of mixed Hodge numbers as before, but on the cohomology with local coefficients in $L_{B, \gamma}$.

Note that when $\gamma=1$, the flat line bundle $L_{B, \gamma}$ is equivariantly trivial. So we can regard the formula as saying

$$
E_{\mathrm{st}}^{B}(M / \Gamma)=E(M)^{\Gamma}+\cdots
$$

where the dots denote the "higher terms" obtained from the fixed points of $\gamma \neq 1$. In particular, viewing a smooth $M$ as $M /\{1\}$, we find that $E_{\mathrm{st}}^{B}(M)=E(M)$ for any flat gerbe $B$. That is, the $B$-field affects the Hodge numbers only in the singular case.

The case where $B$ is pulled back from a point is already nontrivial. Indeed, we saw in that $\Gamma$-equivariant gerbes on a point are classified up to isomorphism by the discrete torsion 
$H^{2}(\Gamma, \mathrm{U}(1))$. For such a gerbe $B$, each bundle $L_{B, \gamma}$ is trivial and hence is determined by a U(1)-representation of $C(\gamma)$. This turns out to be $\delta \mapsto \nu(\delta, \gamma) / \nu(\gamma, \delta)$, where $\nu$ is any group cocycle representing $B$. The stringy $E$-polynomial therefore agrees in this case with the one defined by Ruan [33]. But we will never use this fact. Our gerbes are not generally pulled back from a point, and in any case we will construct the line bundles $L_{B, \gamma}$ directly. It does so happen, though, that we get the same answer as we would from a certain element of discrete torsion (cf. [22]).

\section{The main conjecture}

Our purpose is to study the stringy mixed Hodge numbers of the moduli spaces $M_{\mathrm{DR}}^{d}\left(\mathrm{SL}_{r}\right)$ and $M_{\mathrm{DR}}^{d}\left(\mathrm{PGL}_{r}\right)$. We will assume, now and henceforth, that $r$ and $d$ are coprime. Since $M_{\mathrm{DR}}^{d}\left(\mathrm{SL}_{r}\right)$ is $\Gamma$-equivariantly diffeomorphic to $M_{\mathrm{Dol}}^{d}\left(\mathrm{SL}_{r}\right)$, we may regard $M_{\mathrm{DR}}^{d}\left(\mathrm{SL}_{r}\right)$ and $M_{\mathrm{DR}}^{d}\left(\mathrm{PGL}_{r}\right)$ as being equipped with the flat unitary gerbes $B$ and $\hat{B}$ defined in $\hat{s}$. We then conjecture the following.

(5.1) Conjecture. For all $d, e \in \mathbb{Z}$ coprime to $r$,

$$
E_{\mathrm{st}}^{B^{e}}\left(M_{\mathrm{DR}}^{d}\left(\mathrm{SL}_{r}\right)\right)=E_{\mathrm{st}}^{\hat{B}^{d}}\left(M_{\mathrm{DR}}^{e}\left(\mathrm{PGL}_{r}\right)\right) .
$$

Since $M_{\mathrm{DR}}^{d}\left(\mathrm{SL}_{r}\right)$ is smooth, the left-hand side actually equals $E\left(M_{\mathrm{DR}}^{d}\left(\mathrm{SL}_{r}\right)\right)$, which of course is independent of $e$.

The rest of the paper is devoted to proving this conjecture for $r=2$ and 3. In fact much of what we prove is valid for general $r$. The broad outline of the argument is as follows.

First, we show in 86 that the stringy Hodge numbers of the De Rham and Dolbeault spaces are the same. Thereafter we may work exclusively with the Dolbeault space, which has the advantage of admitting a $\mathbb{C}^{\times}$-action. So we wish to show

$$
E\left(M_{\mathrm{Dol}}^{d}\left(\mathrm{SL}_{r}\right)\right)=E_{\mathrm{st}}^{\hat{B}^{d}}\left(M_{\mathrm{Dol}}^{e}\left(\mathrm{PGL}_{r}\right)\right) .
$$

In fact both sides are cumbersome to write down in full due to the presence of a complicated "leading term" $E\left(M_{\mathrm{Dol}}^{e}\left(\mathrm{SL}_{r}\right)\right)^{\Gamma}$ : the part invariant under the $\Gamma$-action. But the remaining terms are more tractable. So we will actually subtract it off and verify that

$$
E\left(M_{\text {Dol }}^{d}\left(\mathrm{SL}_{r}\right)\right)-E\left(M_{\mathrm{Dol}}^{e}\left(\mathrm{SL}_{r}\right)\right)^{\Gamma}=E_{\mathrm{st}}^{\hat{B}^{d}}\left(M_{\mathrm{Dol}}^{e}\left(\mathrm{PGL}_{r}\right)\right)-E\left(M_{\mathrm{Dol}}^{e}\left(\mathrm{SL}_{r}\right)\right)^{\Gamma} .
$$

To compute the right-hand side, we need to know about the fixed-point set of the $\Gamma$ action. This is described in $\delta$, and the computation is carried out for $r$ prime in $\S 8$. To compute the left-hand side, we need to know about the fixed-point set of the $\mathbb{C}^{\times}$-action. This is described in 89 , and the computation is carried through far enough to settle the cases $r=2$ and 3 in $\$ 10$. 


\section{Equality of $\boldsymbol{E}_{\mathrm{st}}$-polynomials of the Dolbeault and de Rham spaces}

For brevity, in this section $M_{\mathrm{Hod}}^{d}\left(\mathrm{SL}_{r}\right)$ and $M_{\mathrm{Hod}}^{d}\left(\mathrm{PGL}_{r}\right)$ will be denoted simply by $M_{\text {Hod }}$ and $\hat{M}_{\text {Hod }}$, respectively, and likewise for the Dolbeault and de Rham spaces.

(6.1) Lemma. There exists a proper family $\bar{M}_{\text {Hod }} \rightarrow \mathbb{C}$ containing a divisor $X \times \mathbb{C} \rightarrow \mathbb{C}$ whose complement is $M_{\mathrm{Hod}}$. It is a smoothly trivial family of orbifolds in the sense that it is an orbifold, diffeomorphic to an orbifold times $\mathbb{C}$.

Proof. As seen in 8 园, $\mathbb{C}^{\times}$acts on $M_{\text {Hod }}$ over the action on the base $\mathbb{C}$ by scalar multiplication. Let $\mathbb{C}^{\times}$also act on $\mathbb{C}^{2}$ by $t \cdot(x, y)=(t x, y)$. Then $(x, y) \mapsto x y$ is a $\mathbb{C}^{\times}$-equivariant map $\mathbb{C}^{2} \rightarrow \mathbb{C}$. Let $M^{\prime}$ be the base change of $M_{\text {Hod }}$ given by pulling back by this map; then $\mathbb{C}^{\times}$ acts on $M^{\prime}$. Regarded as a scheme over the second factor $\mathbb{C}$, the fiber of $M^{\prime}$ over $y \neq 0$ is $M_{\text {Hod }}$, but the fiber over $y=0$ is $M_{\text {Dol }} \times \mathbb{C}$, with the diagonal action of $\mathbb{C}^{\times}$.

For any $p \in M^{\prime}$, the limit $\lim _{t \rightarrow 0} t \cdot p$ exists by Corollary 10.5 of Simpson [37]. Moreover, the fixed-point set is $M_{\text {Dol }}^{\mathbb{C}^{\times}} \times \mathbb{C} \rightarrow\{0\} \times \mathbb{C}$, which is proper over $\mathbb{C}$ by Lemma 10.6 of Simpson [37. The hypotheses of Theorem 11.2 of Simpson [37] therefore hold, implying that the open set $U \subset M^{\prime}$ of those $p \in M^{\prime}$ having no $\lim _{t \rightarrow \infty} t \cdot p$ has a geometric quotient, proper and separated over $\mathbb{C}$. This open set is the complement of $\mathcal{N} \times \mathbb{C} \subset M_{\text {Dol }} \times \mathbb{C} \rightarrow\{0\} \times \mathbb{C}$, where $\mathcal{N}$ is the so-called nilpotent cone in $M_{\text {Dol }}$, the zero fiber of the Hitchin map. The quotient $U / \mathbb{C}^{\times}$is the disjoint union of two pieces: an open set is the quotient of $M^{\prime} \backslash\left(M_{\text {Dol }} \times \mathbb{C}\right) \cong$ $M_{\text {Hod }} \times \mathbb{C}^{\times}$, which of course is just $M_{\text {Hod }}$. The remainder is the quotient of $\left(M_{\text {Dol }} \backslash \mathcal{N}\right) \times \mathbb{C}$, which is of the form $X \times \mathbb{C}$, where $X$ is the geometric quotient of $M_{\text {Dol }} \backslash \mathcal{N}$.

Hence the quotient is a proper family of schemes over $\mathbb{C}$ whose nonzero fiber is a compactification of $M_{\mathrm{DR}}$ by adding $X$ as a divisor at infinity, and whose zero fiber is a compactification of $M_{\text {Dol }}$ by adding $X$ as a divisor at infinity. In fact these compactifications are precisely those constructed by Simpson [37] and the first author [20], respectively.

Certainly $\bar{M}_{\text {Hod }}$ is an orbifold, as a geometric quotient of a smooth variety by a $\mathbb{C}^{\times}$action with finite stabilizers. A neighborhood of any point in the zero fiber is diffeomorphic to a trivial family of orbifolds: just note that $\mathbb{C}^{\times}$acts trivially on the base $\mathbb{C}$ and use the usual local model. Then the standard argument showing that a submersion of compact manifolds is locally trivial applies in this orbifold situation: choose a Riemannian metric and flow in a perpendicular direction to the projection. So the family is smoothly trivial in an analytical neighborhood of the zero fiber. But the $\mathbb{C}^{\times}$-action can be used to retract all of $\bar{M}_{\text {Hod }}$ into this neighborhood.

(6.2) Theorem. For $r$ and $d$ coprime, $E\left(M_{\mathrm{Dol}}\right)=E\left(M_{\mathrm{DR}}\right)$.

Proof. The family constructed in the lemma above is a family of compact "rational homology manifolds" in the sense of Deligne [13, (8.2.4)]. The mixed Hodge structures of the fibers are therefore pure, that is, $H^{p, q ; k}=0$ unless $p+q=k$, and Poincaré duality identifies the mixed Hodge structures on the ordinary and compactly supported cohomology. Because of the topological triviality, the restriction from $\bar{M}_{\text {Hod }}$ to any fiber is an isomorphism on 
cohomology, and hence an isomorphism of mixed Hodge structures [12, 3.2.5]. Hence the mixed Hodge structures of $H_{\mathrm{cpt}}^{*}\left(\bar{M}_{\mathrm{Dol}}\right)$ and $H_{\mathrm{cpt}}^{*}\left(\bar{M}_{\mathrm{DR}}\right)$ are isomorphic, and so $E\left(\bar{M}_{\mathrm{Dol}}\right)=$ $E\left(\bar{M}_{\mathrm{DR}}\right)$. But $\bar{M}_{\mathrm{Dol}}$ is a disjoint union $M_{\mathrm{Dol}} \cup X$, while $\bar{M}_{\mathrm{DR}}$ is a disjoint union $M_{\mathrm{DR}} \cup X$. Since the $E$-polynomial is additive under disjoint union, it follows that $E\left(M_{\mathrm{Dol}}\right)=E\left(M_{\mathrm{DR}}\right)$.

(6.3) Lemma. For any $\gamma \in \Gamma, \bar{M}_{\text {Hod }}^{\gamma}$ is a smoothly trivial family of orbifolds with $\bar{M}_{\text {Hod }}^{\gamma} \cap(X \times \mathbb{C})=X^{\gamma} \times \mathbb{C}$.

Proof. The whole argument of Lemma (6.1) goes through provided that $M_{\mathrm{Hod}}^{\gamma}$ is the geometric quotient by $\mathbb{C}^{\times}$of $U^{\gamma}$, where $U \subset M^{\prime}$ is the open set in the proof of Lemma (6.1). In other words, we want to know that $\left(U / \mathbb{C}^{\times}\right)^{\gamma}=U^{\gamma} / \mathbb{C}^{\times}$. This means that if a $\mathbb{C}^{\times}$-orbit is preserved by $\gamma$, then it is fixed pointwise. This is obvious if the orbit does not lie over the $y$-axis in $\mathbb{C}^{2}$, since $\Gamma$ acts trivially on $\mathbb{C}^{2}$ while $\mathbb{C}^{\times}$acts by $t \cdot(x, y)=(t x, y)$. On the other hand, the part of $U$ lying over any point on the $y$-axis is $M_{\text {Dol }} \backslash \mathcal{N}$, the complement of the zero fiber of the Hitchin map. But the Hitchin map $\mu: M_{\text {Dol }} \rightarrow V_{r}$ takes the $\mathbb{C}^{\times}$-action on $M_{\text {Dol }}$ to a linear action on the vector space $V_{r}$ with positive weights, while it takes the $\gamma$-action to the trivial action on $V_{r}$. So the only way for a $\mathbb{C}^{\times}$-orbit outside the zero fiber to be preserved by $\gamma$ is to be fixed pointwise.

(6.4) Theorem. For any $e \in \mathbb{Z}$,

$$
E_{\mathrm{st}}^{\hat{B}^{e}}\left(\hat{M}_{\mathrm{DR}}\right)=E_{\mathrm{st}}^{\hat{B}^{e}}\left(\hat{M}_{\mathrm{Dol}}\right) .
$$

Proof. Both sides are sums over $\gamma \in \Gamma$ by definition; it will be shown that the terms agree, that is,

$$
E_{\mathrm{st}}\left(\hat{M}_{\mathrm{DR}}^{\gamma}, L_{B^{e}, \gamma}\right)=E_{\mathrm{st}}\left(\hat{M}_{\mathrm{Dol}}^{\gamma}, L_{B^{e}, \gamma}\right) .
$$

(The equality of the fermionic shifts is clear since the representations of the finite group $\Gamma$ are rigid.)

We wish to argue as in the proof of Theorem (6.2), but first we need to show that $L_{B^{e}, \gamma} \rightarrow M_{\text {Hod }}^{\gamma}$ extends over $\bar{M}_{\text {Hod }}^{\gamma}$ as a $\Gamma$-equivariant flat line bundle. Since $\bar{M}_{\text {Hod }}$ is constructed in the proof of Lemma $(6 \mathbf{6 . 1})$ as a geometric quotient of the open set $U \subset M^{\prime}$ described there, for this it suffices to establish two statements: first, that the $\Gamma$ - and $\mathbb{C}^{\times}$actions on $L_{B^{e}, \gamma}$ commute, and second, that the isotropy of the $\mathbb{C}^{\times}$-action on $U$ acts trivially on $L_{B^{e}, \gamma}$.

The first statement is easy: just notice that since $B^{e}$ is a $\mathbb{Z}_{r}$-gerbe, $L_{B^{e}, \gamma}$ has disconnected structure group $\mathbb{Z}_{r}$, whereas $\mathbb{C}^{\times}$is connected. Since $1 \in \mathbb{C}^{\times}$certainly commutes with the $\Gamma$-action, the whole of $\mathbb{C}^{\times}$must.

As for the second statement, note that for any $p \in U$, the isotropy group of the limit $\lim _{t \rightarrow 0} t \cdot p \in M^{\prime}$ is $\mathbb{C}^{\times}$, and by the same connectedness argument as in the previous paragraph this isotropy group acts trivially on $L_{B^{e}, \gamma}$. Hence by continuity the isotropy groups of $t \cdot p$, even though they may be disconnected, must also act trivially. 
Now proceed as in the proof of Theorem (6.2), using the mixed Hodge structure on cohomology with local coefficients [1, 41]. Lemma (6.3) guarantees that the same scheme $X^{\gamma}$ gets added at infinity to compactify both the De Rham and the Dolbeault fibers.

\section{Fixed points of the $\Gamma$-action}

The action of $\Gamma$ on the moduli space of stable bundles was studied in a wonderful paper of Narasimhan and Ramanan [30]. The arguments in $\S 3$ of their paper carry over without change to the space of Higgs bundles.

Fix $\gamma \in \Gamma$ and let $m$ be its order. Let $\pi: \tilde{C} \rightarrow C$ be the unbranched cyclic cover consisting of the $m$ th roots of unity in the total space of $L_{\gamma}$. A bundle on $C$ is equivalent to a $\mathbb{Z}_{m}$-equivariant bundle on $\tilde{C}$, where $\mathbb{Z}_{m}$ is the Galois group.

Let $(\mathbf{E}, \boldsymbol{\Phi})$ be a universal Higgs bundle on $\tilde{M}_{\text {Dol }}^{d}\left(\mathrm{GL}_{r / m}\right) \times \tilde{C}$, where the tilde denotes a moduli space of bundles on $\tilde{C}$. Then $\boldsymbol{\Phi}$ induces a Higgs field on $\pi_{*} \mathbf{E}$; call it $\pi_{*} \boldsymbol{\Phi}$, and regard $\left(\pi_{*} \mathbf{E}, \pi_{*} \boldsymbol{\Phi}\right)$ as a family of Higgs bundles on $C$ parametrized by $\tilde{M}_{\text {Dol }}^{d}\left(\mathrm{GL}_{r / m}\right)$. More precisely, note that as families of $\mathbb{Z}_{m}$-equivariant bundles on $\tilde{C}$

$$
\pi^{*} \pi_{*} \mathbf{E} \cong \bigoplus_{i=1}^{m}\left(1 \times \xi^{i}\right)^{*} \mathbf{E},
$$

where $\xi$, the standard generator of $\mathbb{Z}_{m}$, acts on the right-hand side by cyclically permuting the factors; then the block-diagonal Higgs field $\bigoplus_{i=1}^{m}\left(1 \times \xi^{i}\right)^{*} \boldsymbol{\Phi}$ on the right-hand side descends to the Higgs field we have called $\pi_{*} \boldsymbol{\Phi}$. There is therefore an induced morphism $\tilde{M}_{\text {Dol }}^{d}\left(\mathrm{GL}_{r / m}\right) \rightarrow M_{\text {Dol }}^{d}\left(\mathrm{GL}_{r}\right)$. Moreover, if $\delta \in \Gamma$ acts on $\tilde{M}_{\text {Dol }}^{d}\left(\mathrm{GL}_{r / m}\right)$ by tensorization by $\pi^{*} L_{\delta}$, then this morphism is $\Gamma$-equivariant.

(7.1) Proposition. The action of $\mathbb{Z}_{m}$ on $\tilde{M}_{\mathrm{Dol}}^{d}\left(\mathrm{GL}_{r / m}\right)$ is free, and the morphism to $M_{\mathrm{Dol}}^{d}\left(\mathrm{GL}_{r}\right)$ induced by $\left(\pi_{*} \mathbf{E}, \pi_{*} \boldsymbol{\Phi}\right)$ descends to a $\Gamma$-equivariant regular embedding

$$
\tilde{M}_{\text {Dol }}^{d}\left(\mathrm{GL}_{r / m}\right) / \mathbb{Z}_{m} \rightarrow M_{\text {Dol }}^{d}\left(\mathrm{GL}_{r}\right)
$$

whose image is the fixed-point set $M_{\text {Dol }}^{d}\left(\mathrm{GL}_{r}\right)^{\gamma}$.

Proof. This proposition is analogous to Proposition 3.3 of Narasimhan-Ramanan, and the proof is entirely similar. The open set $U$ that appears in their statements is unnecessary for us, since we are assuming that $r$ and $d$ are coprime.

Our next task is to "fix the determinant," that is, pass from structure group $\mathrm{GL}_{r}$ to $\mathrm{SL}_{r}$. This requires some basic facts about the Prym variety of an unbranched cyclic cover. The proofs of the following are pleasant exercises, and copious hints can be found in Arbarello et al. [2, Appendix B2].

- Let $\tilde{J}^{d}=\operatorname{Pic}^{d} \tilde{C}$ and $J^{d}=\operatorname{Pic}^{d} C$. Then the kernel of $\pi^{*}: J^{0}[m] \rightarrow \tilde{J}^{0}[m]$ is generated by $\gamma$ [Exercise 14 in Arbarello et al.]. 
- The kernel of the norm map Nm : Pic $\tilde{C} \rightarrow \operatorname{Pic} C$ has $m$ components [Exercise 19]. Call the identity component the Prym variety $P$.

- The map Pic $\tilde{C} \rightarrow \operatorname{ker} \mathrm{Nm}$ given by $L \mapsto L^{-1} \otimes \xi^{*} L$, where $\xi \in \mathbb{Z}_{m}$ is the standard generator, is surjective [Exercise 20].

- For $\delta \in J^{0}[m], \pi^{*} L_{\delta}$ is in the image of $\tilde{J}^{d}$ if and only if $\langle\gamma, \delta\rangle=\xi^{d}$, where $\langle$,$\rangle is the$ Weil pairing or intersection form on $J^{0}[m]=H_{1}\left(C, \mathbb{Z}_{m}\right)$ [Exercise 23].

- For $L \in J^{d}$ with $(m, d)=1$, the Galois group $\mathbb{Z}_{m}$ of $\tilde{C} \rightarrow C$ acts transitively on the set of components of $\mathrm{Nm}^{-1}(L)$ [30, Proposition 3.5].

There are natural splittings $M_{\text {Dol }}^{d}\left(\mathbb{C}^{\times}\right)=\operatorname{Pic}^{d} C \times H^{0}(C, K)$ and $\tilde{M}_{\text {Dol }}^{d}\left(\mathbb{C}^{\times}\right)=\operatorname{Pic}^{d} \tilde{C} \times$ $H^{0}(\tilde{C}, K)$. Define $\Pi: \tilde{M}_{\text {Dol }}^{d}\left(\mathbb{C}^{\times}\right) \rightarrow M_{\text {Dol }}^{d}\left(\mathbb{C}^{\times}\right)$to be $\operatorname{det} \pi_{*}$ on the first factor and the obvious sum map on the second factor. It is easy to see that det $\pi_{*}$ equals $\mathrm{Nm}$ if $m$ is odd, and $\mathrm{Nm}$ composed with tensorization by $L_{\gamma}^{m / 2}$ if $m$ is even: see Narasimhan-Ramanan for details. Hence the fibers of $\Pi$ are torsors for $T^{*}$ ker $\mathrm{Nm}$ (over a point).

(7.2) Lemma. The map induced by $\left(\pi_{*} \mathbf{E}, \pi_{*} \boldsymbol{\Phi}\right)$ lies in the following commutative diagram:

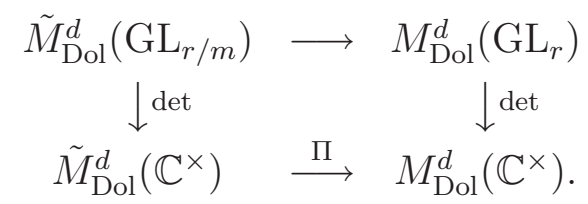

Proof. This is analogous to Lemma 3.4 of Narasimhan-Ramanan.

(7.3) Corollary. If $m=r$, then the fixed-point set $M_{\text {Dol }}^{d}\left(\mathrm{SL}_{r}\right)^{\gamma}$ is the quotient by $\mathbb{Z}_{m}$ of $\Pi^{-1}(L, 0)$ for $L \in J^{d}$, which can be identified with a connected component of $\Pi^{-1}(L, 0)$, or with the total space of the cotangent bundle of $\mathrm{Nm}^{-1}(L)$. It is a torsor for $T^{*} P$ (over a point).

Proof. Since by definition $M_{\text {Dol }}^{d}\left(\mathrm{SL}_{r}\right)=\operatorname{det}^{-1}(L, 0)$, this follows immediately from the lemma and the facts preceding it.

The identification in the corollary above is certainly convenient, but it complicates the $\Gamma$-action slightly. Tensorization by a line bundle of the form $\pi^{*} L_{\delta}$ may interchange the components of the fiber of $\pi$, and we then have to act by an element of $\mathbb{Z}_{m}$ to get back into our chosen one. The following result clarifies how this works.

(7.4) Proposition. (i) Let $L \in J^{d}$ where $(m, d)=1$, and let $q \in \mathbb{Z}$ satisfy $q d \equiv$ $1(\bmod m)$. Then the action by tensorization of $J^{0}[m]$ on $\mathrm{Nm}^{-1}(L)$ is transitive on the set of components, and $\delta \in J^{0}[m]$ acts on the components in the same way as $\langle\gamma, \delta\rangle^{q} \in \mathbb{Z}_{m}$. (ii) The Galois group $\mathbb{Z}_{m}$ acts on the Lie algebra of the Prym as $g-1$ copies of the regular representation of $\mathbb{Z}_{m}$ minus its trivial factor. 


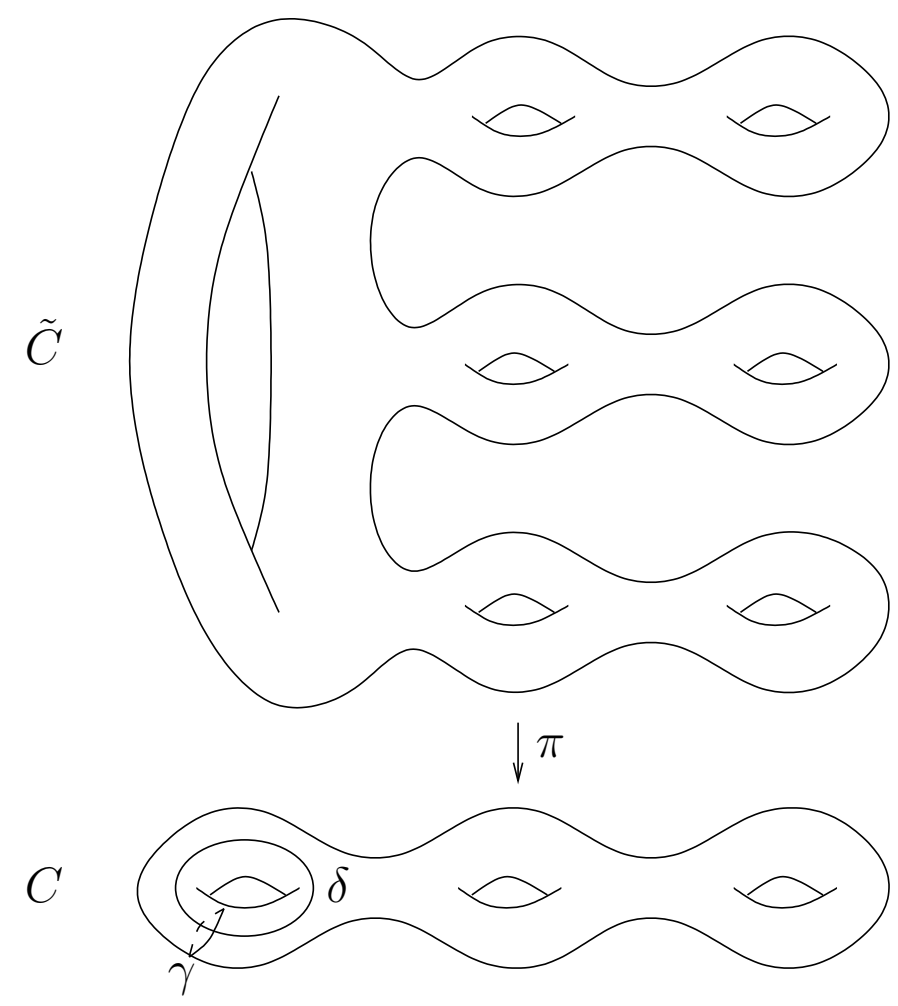

Proof. The most enjoyable proof is topological. Identify $J^{0}[m]$ with $H_{1}\left(C, \mathbb{Z}_{m}\right)$; then Nm corresponds to the push-forward, and $\pi^{*}$ to the pullback of the Poincare dual or inverse image, which we denote by $\pi^{-1}$. Since the intersection form is nondegenerate and $\gamma$ has order $m$, one can choose a set of generators for $H_{1}\left(C, \mathbb{Z}_{m}\right)$ starting with $\gamma$ so that the intersection form is standard. Consequently, there exists a handle presentation of $C$ so that $\gamma$ is represented by a loop around the first handle. The cover $\tilde{C}$ can then be depicted as in the diagram.

The map whose image is the Prym is $L \mapsto L^{-1} \otimes \xi^{*} L$; its restriction to $\tilde{J}^{0}[m]=H_{1}\left(\tilde{C}, \mathbb{Z}_{m}\right)$ is better expressed in additive notation as $a \mapsto \xi^{-1}(a)-a$. So we need to find an element $\delta \in H_{1}\left(C, \mathbb{Z}_{m}\right)$ such that neither $\pi^{-1}(\delta)$ nor any of its nonzero multiples are in the image of this map.

The loop marked $\delta$ on the diagram clearly satisfies this requirement. On the other hand, the inverse images of all loops on the last $g-1$ handles, equally clearly, are in the image of this map. So the powers of $\delta$ act transitively on the set of components, and the loops on the last $g-1$ handles act trivially. Since $\langle\gamma, \delta\rangle=\xi \in \mathbb{Z}_{m}$ it now suffices for (i) to show that $\delta$ acts as $\xi^{q}$. Since the $\Gamma$ - and $\mathbb{Z}_{m}$-actions clearly commute, $\delta$ must act as some power of $\xi$. But according to the penultimate basic fact, there exists $M \in \tilde{J}^{1}$ such that $M^{-1} \otimes \xi^{-1} M \cong \pi^{*} L_{\delta}$, and hence $\xi^{*} M^{d} \cong \pi^{*} L_{d \delta} \otimes M^{d}$. Tensor $M$ by a line bundle pulled back from $J^{0}$ so as to arrange that $M^{d} \in \mathrm{Nm}^{-1}(L)$. Then the actions of $\xi$ and $d \delta$ agree on the component containing $M^{d}$, and hence on all components. Therefore the same is true of $\xi^{q}$ and $\delta$. This proves (i). 
The Lie algebra of the Prym can be identified with $H^{1}(\tilde{C}, \mathbf{R}) / H^{1}(C, \mathbf{R})$. This is spanned by the loops on the last $m(g-1)$ handles of $\tilde{C}$, modulo the inverse images of the loops from the last $g-1$ handles of $C$, and (ii) is now clear.

(7.5) Corollary. Let $L$ and $q$ be as above, and identify $M_{\text {Dol }}^{d}\left(\mathrm{SL}_{r}\right)^{\gamma}$ with the cotangent bundle of a connected component of $\mathrm{Nm}^{-1}(L)$. Then the $\Gamma$-action on $M_{\mathrm{Dol}}^{d}\left(\mathrm{SL}_{r}\right)^{\gamma}$ is induced by the following action on that connected component: $\delta \in \Gamma$ acts by tensorization by $\pi^{*} L_{\delta}$ followed by the action of $\langle\gamma, \delta\rangle^{-q} \in \mathbb{Z}_{m}$. This acts on $H^{1}\left(M_{\mathrm{Dol}}^{d}\left(\mathrm{SL}_{r}\right)^{\gamma}, \mathbf{R}\right)$ as stated in (ii) above.

\section{Calculation for $\mathrm{PGL}_{r}$}

Suppose that $r$ is prime and that $d$ and $e$ are coprime to $r$. Then we can work out the righthand side of (5.2) completely. By abuse of notation we refer henceforth to the fixed-point set $M_{\mathrm{Dol}}^{d}\left(\mathrm{SL}_{r}\right)^{\gamma}$ as $T^{*} P$, although it is really a torsor for $T^{*} P$ over a point.

(8.1) Proposition. The $\Gamma$-equivariant flat line bundle $L_{B, \gamma} \rightarrow T^{*} P$ is trivial, and the $\Gamma$-action is given by the character $\delta \mapsto\langle\gamma, \delta\rangle^{-q}$, where $\mathbb{Z}_{r}$ is identified as usual with the complex $r$ th roots of unity.

Proof. Since we are studying a flat line bundle, instead of working with $T^{*} P$ we may work just with the zero section. This is convenient, since the Higgs field vanishes there, so we may forget it and think of the universal family as merely a bundle.

We abusively call the zero section $P$, but it is really a component of $\mathrm{Nm}^{-1}(L) \subset \tilde{J}^{d}$, which is a torsor for $P$. According to Corollary (7.5),$\delta \in \Gamma$ acts on it by tensorization by $\pi^{*} L_{\delta}$ followed by the action of $\langle\gamma, \delta\rangle^{-q} \in \mathbb{Z}_{m}$. We now explain how to lift both of these actions to (projective) actions on the universal bundle.

First, let $\xi=e^{2 \pi i / m} \in \mathbb{Z}_{m}$ act as an element of the Galois group, both on $\mathrm{Nm}^{-1}(L) \subset \tilde{J}^{d}$ and on $\tilde{C}$ itself. Take a universal line bundle $\mathcal{L} \rightarrow \tilde{J}^{d} \times \tilde{C}$; then by the universal property, $(\xi \times \xi)^{*} \mathcal{L} \cong Q \otimes \mathcal{L}$ for some line bundle $Q \rightarrow \tilde{J}^{d}$.

The push-forward $\pi_{*} \mathcal{L}$ is the desired universal bundle. Since $\pi: \tilde{C} \rightarrow C$ is a Galois cover, there is an isomorphism of $\mathbb{Z}_{m}$-equivariant bundles

$$
\pi^{*} \pi_{*} \mathcal{L} \cong \bigoplus_{i=1}^{m}\left(1 \times \xi^{i}\right)^{*} \mathcal{L}
$$

where on the right-hand side the factors are cyclically permuted by the action.

Hence there are isomorphisms of $\mathbb{Z}_{m}$-equivariant bundles

$$
(\xi \times 1)^{*} \pi^{*} \pi_{*} \mathcal{L} \cong \bigoplus_{i=1}^{m}\left(\xi \times \xi^{i}\right)^{*} \mathcal{L}
$$




$$
\begin{aligned}
& \cong \bigoplus_{i=1}^{m}\left(1 \times \xi^{i-1}\right)^{*}(\xi \times \xi)^{*} \mathcal{L} \\
& \cong \bigoplus_{j=1}^{m}\left(1 \times \xi^{j}\right)^{*}(Q \otimes \mathcal{L}), \\
& \cong Q \otimes \pi^{*} \pi_{*} \mathcal{L},
\end{aligned}
$$

where the penultimate step makes the change of variables $j=i-1$. This descends to the desired isomorphism $(\xi \times 1)^{*} \pi_{*} \mathcal{L} \cong Q \otimes \pi_{*} \mathcal{L}$.

Restricting to $P \times\{c\}$, we find that the projective bundle $\left.\Psi\right|_{P}$ involved in the definition of the gerbe $\left.B\right|_{P}$ is in fact the projectivization of the vector bundle

$$
V=\bigoplus_{y \in \pi^{-1}(c)} \mathcal{L}_{y}
$$

where $\mathcal{L}_{y}=\left.\mathcal{L}\right|_{P \times\{y\}}$, and that the projective action of $\xi$ cyclically permutes the summands.

Second, let $T_{\delta}: \tilde{J}^{d} \rightarrow \tilde{J}^{d}$ denote tensorization by $\pi^{*} L_{\delta}$, which preserves $\operatorname{Nm}^{-1}(L)$. If $\mathcal{L}$ is chosen to be trivial over a basepoint in $\tilde{C}$, then by the universal property, $\left(T_{\delta} \times 1\right)^{*} \mathcal{L} \cong$ $\pi^{*} L_{\delta} \otimes \mathcal{L}$. Hence there are isomorphisms of $\Gamma$-equivariant bundles

$$
\begin{aligned}
\left(T_{\delta} \times 1\right)^{*} \pi^{*} \pi_{*} \mathcal{L} & \cong \bigoplus_{i=1}^{m}\left(T_{\delta} \times \xi^{i}\right)^{*} \mathcal{L} \\
& \cong \bigoplus_{i=1}^{m}\left(1 \times \xi^{i}\right)^{*}\left(\pi^{*} L_{\delta} \otimes \mathcal{L}\right) \\
& \cong \pi^{*} L_{\delta} \otimes \bigoplus_{i=1}^{m}\left(1 \times \xi^{i}\right)^{*} \mathcal{L} \\
& \cong \pi^{*} L_{\delta} \otimes \pi^{*} \pi_{*} \mathcal{L}
\end{aligned}
$$

descending to an isomorphism $T_{\delta}^{*} \pi_{*} \mathcal{L} \cong L_{\delta} \otimes \pi_{*} \mathcal{L}$.

Restricting again to $P \times\{c\}$, we find that $T_{\delta}$ acts on $V$ as an isomorphism on each summand. For example, if $\delta=\gamma$, then $\pi^{*} L_{\gamma}$ is the trivial bundle, with $\xi$ acting by multiplication by $e^{2 \pi i / m}$; so for each $y \in \pi^{-1}(c)$, the isomorphism $\mathcal{L}_{\xi \cdot y} \rightarrow \mathcal{L}_{\xi \cdot y}$ is $e^{-2 \pi i / m}$ times the isomorphism $\mathcal{L}_{y} \rightarrow \mathcal{L}_{y}$. In particular, the automorphism of $\left.\Psi\right|_{P}$ induced by the action of $\gamma$ lifts to an automorphism of $V$ : in other words, it takes this lifting to an isomorphic lifting. This means that the flat line bundle $L_{B, \gamma}$ defined by the automorphism of the gerbe of liftings $\left.B\right|_{P}$ is trivial.

However, the action of $\Gamma$ on this flat line bundle is not trivial. Indeed, the action of $\delta$ as described in Corollary (7.5) lifts to a projective action on $\pi_{*} \mathcal{L}$ via the isomorphisms above. This leads to a diagram

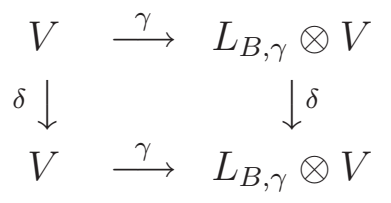


but, to make the diagram commutative, we must multiply $L_{B, \gamma}$ by a scalar factor. Since $\delta$ cyclically permutes the summands as $\langle\gamma, \delta\rangle^{q} \in \mathbb{Z}_{m}$ and $\gamma$ acts on each successive summand as $e^{-2 \pi i / m}$ times the previous one, this factor is $\langle\gamma, \delta\rangle^{-q}$, as desired.

(8.2) Proposition. When $r$ is prime, the right-hand side of (5.2) equals

$$
\frac{1}{r}\left(r^{2 g}-1\right)(u v)^{\left(r^{2}-1\right)(g-1)}\left((1-u)^{(r-1)(g-1)}(1-v)^{(r-1)(g-1)}-\left(\frac{\left(1-u^{r}\right)\left(1-v^{r}\right)}{(1-u)(1-v)}\right)^{g-1}\right) .
$$

Proof. The definition (4.1) of stringy Hodge numbers calls for adding up a contribution from the fixed-point set of each nontrivial $\gamma \in \Gamma$. As seen in Corollary (7.3), this fixed-point set is (a torsor for) $T^{*} P$. The compactly supported cohomology of $T^{*} P \cong \mathbb{C}^{(r-1)(g-1)} \times P$ splits according to the Künneth formula, and that of the first factor is of course $\Gamma$-invariant, so

$$
\begin{aligned}
E\left(T^{*} P, L_{B, \gamma}\right)^{\Gamma} & =E\left(\mathbb{C}^{(r-1)(g-1)}\right) E\left(P, L_{B, \gamma}\right)^{\Gamma} \\
& =(u v)^{(r-1)(g-1)} E\left(P, L_{B, \gamma}\right)^{\Gamma} .
\end{aligned}
$$

To evaluate the right-hand side, note that by Corollary (7.5), any $\delta \in \Gamma$ acts on $H^{1}(P, \mathbf{R})$, and hence on $H^{0,1}(P)$, with eigenvalues $\langle\gamma, \delta\rangle^{k}$, for $k=1$ to $r-1$, each repeated $g-1$ times. Since

$$
H^{k}(P, \mathbb{C})=\Lambda^{k}\left(H^{0,1}(P) \oplus H^{1,0}(P)\right),
$$

as a polynomial with coefficients in the characters of $\Gamma$,

$$
E(P)=\left(\prod_{i=1}^{r-1}\left(1-\rho^{i} u\right)\left(1-\rho^{i} v\right)\right)^{g-1}
$$

where $\rho(\delta)=\langle\gamma, \delta\rangle$, and $E\left(P, L_{B, \gamma}\right)=\rho^{-e} E(P)$. The invariant part is the average value:

$$
\begin{aligned}
E\left(P, L_{B, \gamma}\right)^{\Gamma} & =\frac{1}{|\Gamma|} \sum_{\delta \in \Gamma} \rho^{-1}(\delta)\left(\prod_{i=1}^{r-1}\left(1-\rho^{i}(\delta) u\right)\left(1-\rho^{i}(\delta) v\right)\right)^{g-1} \\
& =\frac{1}{r} \sum_{i=0}^{r-1} \xi^{-e i}\left(\prod_{i=1}^{r-1}\left(1-\xi^{i} u\right)\left(1-\xi^{i} v\right)\right)^{g-1} \\
& =\frac{1}{r}\left((1-u)^{(r-1)(g-1)}(1-v)^{(r-1)(g-1)}-\left(\frac{\left(1-u^{r}\right)\left(1-v^{r}\right)}{(1-u)(1-v)}\right)^{g-1}\right),
\end{aligned}
$$

where $\xi=e^{2 \pi i / r}$.

To compute the fermionic shift, note that $\gamma$ acts with nontrivial weights on the normal bundle to $T^{*} P$ in $M_{\text {Dol }}^{d}\left(\mathrm{SL}_{r}\right)$. The action of $\gamma$ preserves the holomorphic symplectic structure, since on the dense open set in $M_{\text {Dol }}^{d}\left(\mathrm{SL}_{r}\right)$ isomorphic to the cotangent bundle to the moduli space of stable bundles it corresponds to the tautological symplectic structure. Hence every eigenvalue $e^{2 \pi i \alpha}$ is accompanied by an eigenvalue $e^{2 \pi i(1-\alpha)}$, so the fermionic shift is half the rank of the normal bundle, namely $r(r-1)(g-1)$. Summing over the $r^{2 g}-1$ identical terms yields the grand total in the statement. 


\section{Fixed points of the $\mathbb{C}^{\times}$-action}

The Betti numbers of $M_{\text {Dol }}^{d}\left(\mathrm{SL}_{r}\right)$ are computed by Hitchin [24] and Gothen [17] for ranks 2 and 3 respectively, and the $E$-polynomials can be calculated in the same way. But the complete formula is complicated and unilluminating. All we want to know, as was explained in $\$$, is the Hodge polynomial of what we like to call the variant cohomology: the part not invariant under the action of $\Gamma$. This is given by the left-hand side of (5.2).

For convenience, in this section denote $M_{\text {Dol }}^{d}\left(\mathrm{SL}_{r}\right)$ simply by $M_{\text {Dol }}$. To describe its variant cohomology, we shall consider the action of the multiplicative group $\mathbb{C}^{\times}$on $M_{\text {Dol }}$ given by $\lambda \cdot(E, \phi)=(E, \lambda \phi)$. This commutes with the $\Gamma$-action. Let $\mathcal{F}$ be the fixed-point set.

(9.1) Proposition. As polynomials with coefficients in the characters of $\Gamma$,

$$
E\left(M_{\text {Dol }}\right)=(u v)^{\operatorname{dim} M_{\text {Dol }} / 2} E(\mathcal{F}) .
$$

Proof. The $\mathbb{C}^{\times}$-action satisfies the property that for all $x \in M, \lambda \cdot x$ has a limit as $\lambda \rightarrow 0$. This follows directly from the properness of the Hitchin map, since it takes this $\mathbb{C}^{\times}$-action to a linear action on $V$ with positive weights.

Now there is an algebraic version of the Morse stratification called the Białynicki-Birula stratification [8, 40]. Indeed, in this case it is nothing but the Morse stratification for the moment map for the action of $\mathrm{U}(1) \subset \mathbb{C}^{\times}$.

It implies that $M_{\text {Dol }}$ is a $\Gamma$-invariant union of Zariski locally trivial fiber bundles whose fibers are affine spaces, whose bases are the components of $\mathcal{F}$, and whose projections are given by $x \mapsto \lim _{\lambda \rightarrow 0} \lambda \cdot x$. Not only that, the dimension of the affine space is always $\operatorname{dim} M_{\text {Dol }} / 2$. One could prove this directly by looking at the $\mathbb{C}^{\times}$-action on the deformation space [21]. A lazier proof, however, is just to quote Ginzburg's result [15] that the downward flow from each critical set is Lagrangian, and hence has dimension equal to half that of $M_{\text {Dol }}$. The same is therefore true of the upward flow from each point in the critical set, which is the affine space.

The desired formula follows from the additivity of the $E$-polynomial for disjoint unions and its multiplicativity for Zariski locally trivial fibrations [6, 3.4, 3.7].

(9.2) Lemma (Simpson). If $(E, \phi) \in \mathcal{F}$, then there exists a decomposition $E=\bigoplus E_{i}$ with $\phi\left(E_{i}\right) \subset K \otimes E_{i+1}$. Moreover, the ranks and degrees of the $E_{i}$ are locally constant on $\mathcal{F}$.

Proof. Fix $t \in \mathbb{C}^{\times}$which is not a root of unity. If $(E, \phi)$ is to be in $\mathcal{F}$, then there must be an isomorphism $f: E \rightarrow E$ such that $f \phi=t \phi f$. Such an $f$ is unique up to a scalar, since two such maps $f$ and $f^{\prime}$ give rise to an automorphism $f^{-1} f^{\prime}$ of the stable pair $(E, \phi)$, which must be a scalar. The roots of the characteristic polynomial form an $r$-fold cover of $C$ in $C \times \mathbb{C}$, so they and their multiplicities are constant on $C$. This gives a decomposition of $E$ into generalized eigenspaces $E_{\lambda}$, the kernels of $(f-\lambda)^{r}$. These $E_{\lambda}$ constitute subbundles of 
the universal bundle $\mathbf{E}$ restricted to each connected component of $\mathcal{F}$. For locally on $\mathcal{F}, f$ extends to an automorphism of $\left.\mathbf{E}\right|_{\mathcal{F} \times C}$; indeed, the hypercohomology $\mathbf{H}^{0}$ of the two-term complex End $E \rightarrow K \otimes$ End $E$ on $C$ with $f \mapsto f \phi-t \phi f$ is one-dimensional and generated by the $f$ mentioned above, so the hyper-direct image on $\mathcal{F}\left(\mathbf{R}^{0} \pi\right)_{*}($ End $\mathbf{E} \rightarrow K \otimes$ End $\mathbf{E})$ is locally free of rank 1 . Hence the ranks and degrees of the $E_{\lambda}$ are locally constant on $\mathcal{F}$.

Now $(f-t \lambda)^{r} \phi=t^{r} \phi(f-\lambda)^{r}$, so $\phi$ maps the $\lambda$-generalized eigenspace $E_{\lambda}$ to the $\lambda$ generalized eigenspace $E_{t \lambda}$. Since $t$ is not a root of unity, the eigenvalues break up into finite strings $\lambda, t \lambda, \ldots, t^{k} \lambda$, but as stable Higgs bundles are irreducible, there is only one such string.

It will be convenient to refer to the finite sequence $\left(\mathrm{rk} E_{1}, \mathrm{rk} E_{2}, \ldots\right)$ as the type of the component of $\mathcal{F}$ containing $(E, \phi)$. One possibility is the type $(r)$ consisting of a single number only. This means that the Higgs field vanishes, so the corresponding component is simply the moduli space of stable vector bundles of rank $r$ and fixed determinant.

\section{Calculation for $\mathrm{SL}_{r}$}

Now suppose once again that $r$ is prime. We will calculate the contribution to the variant cohomology of the fixed points of type $(1,1, \ldots, 1)$. Then we will show that in ranks 2 and 3 , these are the only nonzero contributors.

(10.1) Proposition. When $r$ is prime, the fixed components of type $(1,1, \ldots, 1)$ contribute to the variant part of $E\left(M_{\text {Dol }}^{d}\left(\mathrm{SL}_{r}\right)\right)$ exactly the polynomial given in Proposition (8.2).

Proof. A Higgs bundle of type $(1,1, \ldots, 1)$ has the form $E=\bigoplus_{i=1}^{r} L_{i}$ with $\phi_{i}: L_{i} \rightarrow$ $L_{i+1} \otimes K$. We assume that the determinant is fixed to be $\mathcal{O}(d c)$ where $c$ is our basepoint, so $\prod L_{i} \cong \mathcal{O}(d c)$. Let $D_{i}$ be the divisor of zeroes of $\phi_{i}$ and $M_{i}=\mathcal{O}\left(D_{i}\right)$. Then $M_{i} \cong$ $L_{i}^{-1} L_{i+1} K$ and so

$$
\prod_{i=1}^{r-1} M_{i}^{i} \cong L_{r}^{r} K^{r(r-1) / 2}(d c) .
$$

Denote $l_{i}=\operatorname{deg} L_{i}$ and $m_{i}=\operatorname{deg} M_{i}$; then $m_{i}=l_{i}-l_{i+1}+2 g-2$, and

$$
\sum_{i=1}^{r-1} i m_{i} \equiv d(\bmod r) .
$$

By the way, this last constraint is accidentally overlooked in the paper of Gothen [17], leading to some incorrect formulas.

A $\mathbb{C}^{\times}$-invariant Higgs bundle is unstable if and only if it is destabilized by a $\mathbb{C}^{\times}$invariant Higgs subbundle: indeed, this follows immediately from the uniqueness of the Harder-Narasimhan stratification for Higgs bundles. Since the only such subbundles are of the form $\bigoplus_{i=k}^{r} L_{i}$, stability is equivalent to

$$
\frac{l_{k}+l_{k+1}+\cdots+l_{r}}{r-k+1}<\frac{d}{r}
$$


for each $k$. It is a simple exercise to show that these inequalities are satisfied if $0 \leq m_{i} \leq$ $2 g-2$. These are the only values that will contribute to the variant cohomology.

Given effective divisors $D_{i}$ whose degrees satisfy (10.3), all that is needed to construct a Higgs bundle of the type described above is a choice of $L_{r}$, which by (10.2) is determined up to multiplication by an $r$ th root of unity, that is, an element of $\Gamma$. Consequently, each type $(1,1, \ldots, 1)$ component of the fixed-point set is a fibered product of the form

$$
N_{m_{1}, \ldots, m_{r}}=\left(\prod_{i=1}^{r-1} S^{m_{i}} C\right) \times_{\mathrm{Pic} \sum i m_{i} C} \operatorname{Pic}^{l_{r}} C,
$$

where the morphism from $\prod_{i=1}^{r-1} S^{m_{i}} C$ is $\left(D_{i}\right) \mapsto \mathcal{O}\left(\sum i D_{i}\right)$ and the morphism from $\operatorname{Pic}^{l_{r}} C$ is $L \mapsto L^{r} K^{r(r-1) / 2}(d c)$.

The terms in the Hodge decomposition, as representations of $\Gamma$, can be computed by pushing forward to $\prod_{i=1}^{r-1} S^{m_{i}} C$ first, as in Hitchin [24] and Gothen [17]. It turns out that

$$
\begin{aligned}
H^{*}\left(N_{m_{1}, \ldots, m_{r}}, \mathbb{C}\right) & =\bigoplus_{\gamma \in \Gamma} H^{*}\left(\prod_{i} S^{m_{i}} C, \bigotimes_{i} \pi_{i}^{*} \mathcal{L}_{\gamma}^{i}\right) \\
& =\bigoplus_{\gamma \in \Gamma} \bigotimes_{i} \Lambda^{m_{i}} H^{1}\left(C, L_{\gamma}^{i}\right),
\end{aligned}
$$

where the right-hand side denotes cohomology with local coefficients, and $\mathcal{L}_{\gamma} \rightarrow S^{m_{1}} C$ is the flat line bundle obtained either by symmetrizing $L_{\gamma} \rightarrow C$ or by pulling back the corresponding flat line bundle over $\mathrm{Pic}^{0} C$ via the Abel-Jacobi map. The variant part consists of the terms where $\gamma \neq 1$. Since $r$ is prime, $L_{\gamma}^{i}$ is then a nontrivial flat bundle for each $i$ from 1 to $r-1$, and hence $H^{1}\left(C, L_{\gamma}^{i}\right)$ has Hodge type $(g-1, g-1)$; indeed, its $(0,1)$ part can be identified with the Dolbeault cohomology of $L_{\gamma}^{i}$ on $C$.

The contribution of $N_{m_{1}, \ldots, m_{r}}$ to the variant part of the $E$-polynomial is therefore

$$
\left(r^{2 g}-1\right)(u v)^{\left(r^{2}-1\right)(g-1)} \underset{\prod t_{i}^{m_{i}}}{\operatorname{Coeff}}\left(\prod_{i}\left(1-t_{i} u\right)\left(1-t_{i} v\right)\right)^{g-1} .
$$

Here the factor of $r^{2 g}-1$ is the number of nontrivial group elements in $\Gamma$, and the power of $u v$ is the contribution of the normal bundle, as described in (9.1). To sum $m_{i}$ from 0 to $2 g-2$ subject to the constraint (10.3), let $\xi=e^{2 \pi i / r}$ and take the average value of this multiplied by $\xi^{-d}$ : that is,

$$
\begin{aligned}
& \frac{1}{r}\left(r^{2 g}-1\right)(u v)^{\left(r^{2}-1\right)(g-1)} \sum_{j=1}^{r} \xi^{-j d}\left(\prod_{i=1}^{r-1}\left(1-\xi^{i j} u\right)\left(1-\xi^{i j} v\right)\right)^{g-1} \\
& \quad=\frac{1}{r}\left(r^{2 g}-1\right)(u v)^{\left(r^{2}-1\right)(g-1)}\left(((1-u)(1-v))^{(r-1)(g-1)}-\left(\frac{\left(1-u^{r}\right)\left(1-v^{r}\right)}{(1-u)(1-v)}\right)^{g-1}\right) .
\end{aligned}
$$

This is indeed the polynomial given in Proposition (8.2). 
(10.4) Lemma. In any rank $r$, the fixed component of type $(r)$ has no variant cohomology.

Proof. This fixed component is the moduli space of stable vector bundles of rank $\mathrm{r}$ and determinant $\mathcal{O}(d c), c \in C$ being our chosen basepoint. So the desired fact is exactly Theorem 1 of Harder-Narasimhan [19], cf. also Newstead [31] and Atiyah-Bott [3].

(10.5) Lemma. In rank 3 , the fixed components of type $(1,2)$ and $(2,1)$ have no variant cohomology.

Proof. Gothen [17] shows that each such fixed component is a smooth family over $\mathrm{Pic}^{0} \mathrm{C}$ whose fiber is the moduli space of stable rank 2 Bradlow pairs with a certain fixed determinant and a fixed Bradlow parameter $\tau$. As such, this family can be obtained, starting from a projective bundle over $\mathrm{Pic}^{0} C$, by a sequence of blow-ups and blow-downs whose centers are projective bundles over $\mathrm{Pic}^{0} C$ times symmetric products of $C$, in the manner prescribed by the second author [39]. Gothen explains how all the spaces in this sequence can be regarded as parametrizing families of (not necessarily stable) rank 3 Higgs bundles, and it follows that $\Gamma$ acts on all the spaces, compatibly with all the morphisms between them. Furthermore, it acts on the projective bundles by bundle maps, and on their bases by translation of the factor $\mathrm{Pic}^{0} \mathrm{C}$ : this is readily apparent from Gothen's description. Consequently, it acts trivially on the cohomology of each projective bundle in the sequence. The standard description of the cohomology of a blow-up (see e.g. Griffiths-Harris [18, p. 605]) implies that, when a finite group $\Gamma$ acts on a smooth $X$ preserving a smooth $Y \subset X$, it acts trivially on the cohomology of the blow-up along $Y$ if and only if it acts trivially on the cohomology of $X$ and of $Y$. Hence $\Gamma$ acts trivially on the cohomology of the fixed component.

(10.6) Theorem. Conjecture (5.1) holds true for $r=2$ and 3.

Proof. First of all, when $r=2$ or 3 , and $d$ and $e$ are both coprime to $r$, there is of course an isomorphism $M_{\text {Dol }}^{d}\left(\mathrm{SL}_{r}\right) \cong M_{\text {Dol }}^{e}\left(\mathrm{SL}_{r}\right)$ : it is given simply by dualizing (in the case when $r=3$ and $d \not \equiv e$ mod 3$)$ and tensorizing by a line bundle of the appropriate degree. Hence we may substitute $d$ for $e$ on the left-hand side of (5.2) with impunity. It is then just a question of studying the variant part of the $E$-polynomial for $M_{\mathrm{Dol}}^{d}\left(\mathrm{SL}_{r}\right)$.

The contribution of the $(1,1)$ or $(1,1,1)$ components to this variant part, given by Proposition (10.1), agrees with the calculation for $\mathrm{PGL}_{r}$ given in Proposition (8.2). The contributions of the remaining components vanish by the two lemmas above.

More generally, for any prime $r$, Conjecture (5.1) would follow from two further conjectures. First, that $E\left(M_{\text {Dol }}^{d}\left(\mathrm{SL}_{r}\right)\right)=E\left(M_{\mathrm{Dol}}^{e}\left(\mathrm{SL}_{r}\right)\right)$ for all $d$ and $e$ coprime to $r$. Second, that no fixed component besides those of type $(1,1, \ldots, 1)$ contributes to the variant cohomology. We hope to return to these conjectures in the future. 


\section{References}

[1] D. Arapura, Hodge theory with local coefficients and fundamental groups of varieties, Bull. Amer. Math. Soc. (N.S.) 20 (1989) 169-172.

[2] E. Arbarello, M. Cornalba, P. Griffiths, and J. Harris, Geometry of algebraic curves, Vol. I, Grundl. der Math. Wiss. 267, Springer, 1985.

[3] M.F. Atiyah and R. Bott, The Yang-Mills equations over Riemann surfaces, Philos. Trans. Roy. Soc. London Ser. A 308 (1982) 523-615.

[4] V.V. Batyrev, Dual polyhedra and mirror symmetry for Calabi-Yau hypersurfaces in toric varieties, J. Algebraic Geom. 3 (1994) 493-535.

[5] V.V. Batyrev and L.A. Borisov, Mirror duality and string-theoretic Hodge numbers, Invent. Math. 126 (1996) 183-203.

[6] V.V. BAtyrev and D. Dais, Strong McKay correspondence, string-theoretic Hodge numbers and mirror symmetry, Topology 35 (1996) 901-929.

[7] M. Bershadsky, A. Johansen, V. Sadov, and C. Vafa, Topological reduction of 4D SYM to 2D $\sigma$-models, Nucl. Phys. B448 (1995) 166-186.

[8] A. BiaŁynicki-Birula, Some theorems on actions of algebraic groups, Ann. Math. 98 (1973) 480-497.

[9] J.-L. Brylinski, Loop spaces, characteristic classes and geometric quantization, Progress in Math. 107, Birkhäuser, 1993.

[10] D.A. Cox and S. Katz, Mirror symmetry and algebraic geometry, Amer. Math. Soc., 1999.

[11] A. CRAW, An introduction to motivic integration, preprint math.AG/9911179.

[12] P. Deligne, Théorie de Hodge II, Inst. Hautes Études Sci. Publ. Math. 40 (1971) 5-57.

[13] P. Deligne, Théorie de Hodge III, Inst. Hautes Études Sci. Publ. Math. 44 (1974) 5-77.

[14] R. Donagi and D. Gaitsgory, The gerbe of Higgs bundles, preprint math.AG/0005132.

[15] V. Ginzburg, The global nilpotent variety is Lagrangian, Duke Math. J. 109 (2001) 511-519.

[16] J. Giraud, Cohomologie non abélienne, Grundl. math. Wiss. 179, Springer, 1971.

[17] P.B. Gothen, The Betti numbers of the moduli space of stable rank 3 Higgs bundles on a Riemann surface, Internat. J. Math. 5 (1994) 861-875.

[18] P. Griffiths and J. Harris, Principles of algebraic geometry, Wiley, 1978.

[19] G. Harder and M.S. Narasimhan, On the cohomology groups of moduli spaces of vector bundles on curves, Math. Ann. 212 (1974/75) 215-248. 
[20] T. Hausel, Compactification of moduli of Higgs bundles, J. Reine Angew. Math. 503 (1998) 169-192.

[21] T. Hausel and M. Thaddeus, On the cohomology ring of the moduli space of Higgs bundles I, preprint math.AG/0003093.

[22] T. Hausel and M. Thaddeus, Examples of mirror partners arising from integrable systems, C. R. Acad. Sci. Paris Sér. I Math. 333 (2001) 313-318.

[23] N.J. Hitchin, Stable bundles and integrable systems, Duke Math. J. 54 (1987) 91-114.

[24] N.J. Hitchin, The self-duality equations on a Riemann surface, Proc. London Math. Soc. (3) 55 (1987) 59-126.

[25] N.J. Hitchin, The moduli space of special Lagrangian submanifolds, Ann. Scuola Norm. Sup. Pisa Cl. Sci. (4) 25 (1997-8) 503-515.

[26] N.J. Hitchin, Lectures on special Lagrangian submanifolds, Winter School on Mirror Symmetry, Vector Bundles and Lagrangian Submanifolds (Cambridge, MA, 1999), 151-182, Amer. Math. Soc., 2001.

[27] G. Karpilovsky, The Schur multiplier, Oxford Univ. Press, 1987.

[28] D. Mumford, Abelian varieties, Oxford Univ. Press, 1970.

[29] D. Mumford, J. Fogarty, and F. Kirwan, Geometric invariant theory, third enlarged edition, Ergebnisse der Math. 34, Springer, 1994.

[30] M.S. Narasimhan and S. Ramanan, Generalised Prym varieties as fixed points, J. Indian Math. Soc. 39 (1975) 1-19.

[31] P.E. Newstead, Characteristic classes of stable bundles over an algebraic curve, Trans. Amer. Math. Soc. 169 (1972) 337-345.

[32] N. Nitsure, Moduli space of semistable pairs on a curve, Proc. London Math. Soc. (3) 62 (1991) 275-300.

[33] Y. RuAn, Discrete torsion and twisted orbifold cohomology, preprint math.AG/0005299.

[34] J.-P. SERre, Algebraic groups and class fields, Grad. Texts in Math. 117, Springer, 1988.

[35] C.T. Simpson, Moduli of representations of the fundamental group of a smooth projective variety, I, Inst. Hautes Études Sci. Publ. Math. 79 (1994) 47-129.

[36] C.T. Simpson, Moduli of representations of the fundamental group of a smooth projective variety, II, Inst. Hautes Études Sci. Publ. Math. 80 (1995) 5-79.

[37] C.T. Simpson, The Hodge filtration on nonabelian cohomology, Algebraic geometry-Santa Cruz 1995, Proc. Symp. Pure Math. 62, ed. J. Kollár, R. Lazarsfeld, and D. Morrison, American Math. Soc., 1997. 
[38] A. Strominger, S.-T. Yau, and E. Zaslow, Mirror symmetry is T-duality, Nuclear Phys. B 479 (1996) 243-259.

[39] M. Thaddeus, Stable pairs, linear systems and the Verlinde formula, Invent. Math. 117 (1994) 317-353.

[40] M. Thaddeus, Geometric invariant theory and flips, J. Amer. Math. Soc. 9 (1996) 691-723.

[41] K. Timmerscheidt, Mixed Hodge theory for unitary local systems, J. Reine Angew. Math. 379 (1987) 152-171.

[42] C. VAfa, String vacua and orbifoldized LG models, Modern Phys. Lett. A 4 (1989) 1169-1185.

[43] C. Vafa and E. Witten, On orbifolds with discrete torsion, J. Geom. Phys. 15 (1995) 189-214.

[44] E. ZAsLOw, Topological orbifold models and quantum cohomology rings, Comm. Math. Phys. 156 (1993) 301-331. 